\title{
A study of third-order single-valued and multi-valued problems with integral boundary conditions
}

\author{
Hamed H Alsulami ${ }^{1 *}$, Sotiris K Ntouyas², Saleh A Al-Mezel ${ }^{1}$, Bashir Ahmad ${ }^{1}$ and Ahmed Alsaedi ${ }^{1}$
}

"Correspondence:

hamed9@hotmail.com

'Department of Mathematics,

Faculty of Science, King Abdulaziz University, P.O. Box 80203, Jeddah, 21589, Saudi Arabia

Full list of author information is available at the end of the article

\begin{abstract}
This paper investigates some nonlinear third-order ordinary differential equations and inclusions with anti-periodic type integral boundary conditions and multi-strip boundary conditions. To establish the existence results for the given problems, we apply standard tools of fixed point theory for single-valued and multi-valued problems. The results are illustrated with the aid of examples.

MSC: 34B10; 34B15; 34A60
\end{abstract}

Keywords: differential equations; differential inclusions; third-order; integral boundary conditions; fixed point

\section{Introduction}

Boundary value problems for nonlinear differential equations arise in a variety of areas of applied mathematics, physics and variational problems of control theory. A point of central importance in the study of nonlinear boundary value problems is to understand how the properties of nonlinearity in a problem influence the nature of the solutions to the boundary value problems. Much of the work on the existence of solutions to the boundary value problems involves second-order differential equations. Third-order differential equations arise in a variety of problems ranging from the study of regularization of the Cauchy problem for one-dimensional hyperbolic conservation laws [1] to nano boundary layer fluid flows [2] or to describe the evolution of physical phenomena in fluctuating environments [3]. Examples include many famous equations in mathematical physics, such as the Korteweg-de Vries equation [4]. Some results concerning the existence of solutions for third-order differential equations can be found in [5-17].

In recent years, computational fluid dynamics (CFD) techniques have been used increasingly by researchers seeking to understand vascular hemodynamics. Most of the CFDbased hemodynamic studies so far have been conducted to represent in vitro conditions within restrictive assumptions. These studies under in vitro conditions are well suited to investigate basic phenomena related to fluid dynamics in vessels models but are not fully representative of actual patient hemodynamic conditions. In fact, CFD methods possess the potential to augment the data obtained from in vitro methods by providing a complete characterization of hemodynamic conditions (blood velocity and pressure as a function of space and time) under precisely controlled conditions. However, specific difficulties in

(c) 2015 Alsulami et al.; licensee Springer. This is an Open Access article distributed under the terms of the Creative Commons Attribution License (http://creativecommons.org/licenses/by/4.0), which permits unrestricted use, distribution, and reproduction in any medium, provided the original work is properly credited. 
CFD studies of blood flows are related to the boundary conditions. It is now recognized that the blood flow in a given district may depend on the global dynamics of the whole circulation. Consequently, it is sometimes necessary to couple the 3D blood flow solver to a low-order model for the entire vascular system [18]. A second difficulty is related to the limitations of the existing in vitro anemometry techniques. Indeed, the space resolution is far too coarse to tackle even the largest scales of the blood flow details. As a consequence, the boundary conditions (e.g. the instantaneous velocity profile at the inlet section of the computed domain) are unknown for an in vitro blood flow computation. Most of the times, one assumes some analytical space-time evolution for prescribing the inlet profile. Taylor et al. [19] propose to assume very long circular vessel geometry upstream the inlet section so that the analytic solution of Womersley [20] can be prescribed. However, it is not always justified to assume a circular cross section. In order to cope with this problem, an alternative approach prescribing integral boundary conditions is presented in [21]. The validity of this approach is verified by computing both steady and pulsated channel flows for Womersley number up to 15 . As a matter of fact, problems with integral boundary conditions arise naturally in thermal conduction problems [22], semiconductor problems [23], hydrodynamic problems [24], etc. Integral boundary conditions have various applications in applied sciences such as blood flow problems, chemical engineering, thermoelasticity, underground water flow, population dynamics, etc. For more details of boundary value problems involving integral boundary conditions; see for instance, [2534] and references therein.

The main tools of our study are fixed point theorems. Several authors have used fixed point theory to show the existence of solutions to boundary value problems. The texts by Agarwal et al. [35] and by Guo and Lakshmikantham [36] are excellent resources for the use of fixed point theory in the study of existence of solutions to boundary value problems.

In this paper, we discuss several new results for third-order boundary value problems of differential equations and inclusions with new kinds of integral boundary conditions. The work accomplished in this paper is important from theoretical as well as from practical point of view. The third-order differential equations occur in a variety of problems of applied sciences such as conservation laws, nano boundary layer fluid, evolution of physical phenomena in fluctuating environments, etc. Therefore the present study will provide a platform for solving these problems by means of analytic/numerical techniques. The paper is organized as follows.

In Section 2, we consider the following problem of third-order differential equations with anti-periodic type integral boundary conditions:

$$
\left\{\begin{array}{l}
x^{\prime \prime \prime}(t)=f(t, x(t)), \quad t \in[0, T], T>0, \\
x(0)-\lambda_{1} x(T)=\mu_{1} \int_{0}^{T} g(s, x(s)) d s, \\
x^{\prime}(0)-\lambda_{2} x^{\prime}(T)=\mu_{2} \int_{0}^{T} h(s, x(s)) d s, \\
x^{\prime \prime}(0)-\lambda_{3} x^{\prime \prime}(T)=\mu_{3} \int_{0}^{T} \phi(s, x(s)) d s,
\end{array}\right.
$$

where $f, g, h, \phi:[0, T] \times \mathbb{R} \rightarrow \mathbb{R}$ are given continuous functions and $\lambda_{j}, \mu_{j} \in \mathbb{R}\left(\lambda_{j} \neq 1, j=\right.$ $1,2,3)$. The existence of solutions is obtained by means of the Leray-Schauder nonlinear alternative, the Banach contraction mapping principle, and the Krasnoselskii fixed point theorem. 
In Section 3, we consider a third-order nonlinear boundary value problem with multistrip boundary conditions given by

$$
\left\{\begin{array}{l}
x^{\prime \prime \prime}(t)=f(t, x(t)), \quad 0<t<1 \\
x(0)=0, \quad x^{\prime}(0)=0, \\
x(1)=\sum_{i=1}^{n-2} \alpha_{i} \int_{\zeta_{i}}^{\eta_{i}} x(s) d s, \quad 0<\zeta_{i}<\eta_{i}<1, i=1,2, \ldots, n-2, n \geq 3
\end{array}\right.
$$

where $f$ is a given continuous function and $\alpha_{i} \in \mathbb{R}$ satisfy the condition:

$$
\sum_{i=1}^{n-2} \alpha_{i}\left(\eta_{i}^{3}-\zeta_{i}^{3}\right) \neq 3
$$

We obtain some new existence results for the problem (1.2) by using a variety of fixed point theorems such as the Banach contraction principle, the Krasnoselskii fixed point theorem, the Leray-Schauder nonlinear alternative, and a fixed point theorem due to Boyd and Wong.

Finally, in Section 4, we study the following third-order nonlinear differential inclusion with multi-strip boundary conditions:

$$
\left\{\begin{array}{l}
x^{\prime \prime \prime}(t) \in F(t, x(t)), \quad 0<t<1, \\
x(0)=0, \quad x^{\prime}(0)=0, \\
x(1)=\sum_{i=1}^{n-2} \alpha_{i} \int_{\zeta_{i}}^{\eta_{i}} x(s) d s, \quad 0<\zeta_{i}<\eta_{i}<1, i=1,2, \ldots, n-2, n \geq 3,
\end{array}\right.
$$

where $F:[0,1] \times \mathbb{R} \rightarrow \mathcal{P}(\mathbb{R})$ is a multi-valued map, $\mathcal{P}(\mathbb{R})$ is the family of all nonempty subsets of $\mathbb{R}$. We establish some existence results for the problem (1.3), when the right hand side is convex as well as non-convex valued. In the first result we use the nonlinear alternative of Leray-Schauder type while in the second result, we shall combine the nonlinear alternative of Leray-Schauder type for single-valued maps with a selection theorem due to Bressan and Colombo for lower semicontinuous multi-valued maps with nonempty closed and decomposable values. The third result relies on the fixed point theorem for contraction multi-valued maps due to Covitz and Nadler.

The methods used in all problems above are standard; however, their exposition in the framework of the given problems is new.

\section{Problem I}

The following lemma plays a pivotal role in obtaining the existence results for the problem (1.1).

Lemma 2.1 For a given $y \in C([0, T], \mathbb{R})$, the unique solution of the equation $x^{\prime \prime \prime}(t)=y(t)$, $t \in[0, T]$ subject to the boundary conditions of $(1.1)$ is given by

$$
\begin{aligned}
x(t)= & \int_{0}^{t} \frac{(t-s)^{2}}{2} y(s) d s-\lambda_{1} \xi_{1} \int_{0}^{T} \frac{(T-s)^{2}}{2} y(s) d s \\
& +\lambda_{2} \eta_{2} \int_{0}^{T}(T-s) y(s) d s+\lambda_{3} \eta_{1} \int_{0}^{T} y(s) d s \\
& -\mu_{1} \xi_{1} \int_{0}^{T} g(s, x(s)) d s+\mu_{2} \eta_{2} \int_{0}^{T} h(s, x(s)) d s+\mu_{3} \eta_{1} \int_{0}^{T} \phi(s, x(s)) d s,
\end{aligned}
$$


where

$$
\begin{aligned}
& \eta_{1}=\xi_{3}\left[-\lambda_{1}\left(\lambda_{2}+1\right) T^{2}+2 \lambda_{2}\left(\lambda_{1}-1\right) t T-\left(\lambda_{1}-1\right)\left(\lambda_{2}-1\right) t^{2}\right] \\
& \eta_{2}=\xi_{2}\left[\lambda_{1} T-\left(\lambda_{1}-1\right) t\right], \\
& \xi_{1}=\frac{1}{\lambda_{1}-1}, \quad \xi_{2}=\frac{1}{\left(\lambda_{1}-1\right)\left(\lambda_{2}-1\right)}, \quad \xi_{3}=\frac{1}{2\left(\lambda_{1}-1\right)\left(\lambda_{2}-1\right)\left(\lambda_{3}-1\right)} .
\end{aligned}
$$

Proof It is well known that the solution of the differential equation $x^{\prime \prime \prime}(t)=y(t)$ can be written as

$$
x(t)=\int_{0}^{t} \frac{(t-s)^{2}}{2} y(s) d s-c_{0}-c_{1} t-c_{2} t^{2}, \quad t \in[0, T],
$$

where $c_{0}, c_{1}, c_{2} \in \mathbb{R}$ are arbitrary constants. Applying the boundary conditions of (1.1), we get

$$
\left\{\begin{array}{l}
\left(\lambda_{1}-1\right) c_{0}+\lambda_{1} T c_{1}+\lambda_{1} T^{2} c_{2}=\mu_{1} \int_{0}^{T} g(t, x(s)) d s+\lambda_{1} \int_{0}^{T} \frac{(T-s)^{2}}{2} y(s) d s \\
\left(\lambda_{2}-1\right) c_{1}+2 \lambda_{2} T c_{2}=\mu_{2} \int_{0}^{T} h(s, x(s)) d s+\lambda_{2} \int_{0}^{T}(T-s) y(s) d s \\
2\left(\lambda_{3}-1\right) c_{3}=\mu_{3} \int_{0}^{T} \phi(s, x(s)) d s+\lambda_{3} \int_{0}^{T} y(s) d s
\end{array}\right.
$$

Solving the system (2.3), we find the values of $c_{0}, c_{1}$ and $c_{2}$. Substituting these values in (2.2), we obtain (2.1).

Next we outline the tools that will be used in the sequel.

Theorem 2.2 (Nonlinear alternative for single-valued maps) Let E be a Banach space, $C$ a closed, convex subset of $E, U$ an open subset of $C$ and $0 \in U$. Suppose that $F: \bar{U} \rightarrow C$ is a continuous, compact (that is, $F(\bar{U})$ is a relatively compact subset of $C$ ) map. Then either

(i) F has a fixed point in $\bar{U}$, or

(ii) there is a $u \in \partial U$ (the boundary of $U$ in $C$ ) and $\lambda \in(0,1)$ with $u=\lambda F(u)$.

Lemma 2.3 (Krasnoselskii's fixed point theorem) Let $M$ be a closed bounded, convex and nonempty subset of a Banach space X. Let $A, B$ be the operators such that

(i) $A x+B y \in M$ whenever $x, y \in M$;

(ii) $A$ is compact and continuous, and

(iii) $B$ is a contraction mapping.

Then there exists $z \in M$ such that $z=A z+B z$.

\subsection{Existence results}

Let $\mathcal{C}=C([0, T], \mathbb{R})$ denotes the Banach space of all continuous functions from $[0, T] \rightarrow \mathbb{R}$ endowed with the usual sup-norm $\|x\|=\sup _{t \in[0, T]}|x(t)|$.

By Lemma 2.1, the problem (1.1) can be transformed to a fixed point problem as $x=\mathcal{F} x$, where $\mathcal{F}: \mathcal{C} \rightarrow \mathcal{C}$ is given by

$$
\begin{aligned}
(\mathcal{F} x)(t)= & \int_{0}^{t} \frac{(t-s)^{2}}{2} f(s, x(s)) d s-\lambda_{1} \xi_{1} \int_{0}^{T} \frac{(T-s)^{2}}{2} f(s, x(s)) d s \\
& +\lambda_{2} \eta_{2} \int_{0}^{T}(T-s) f(s, x(s)) d s+\lambda_{3} \eta_{1} \int_{0}^{T} f(s, x(s)) d s
\end{aligned}
$$




$$
\begin{aligned}
& -\mu_{1} \xi_{1} \int_{0}^{T} g(s, x(s)) d s+\mu_{2} \eta_{2} \int_{0}^{T} h(s, x(s)) d s \\
& +\mu_{3} \eta_{1} \int_{0}^{T} \phi(s, x(s)) d s, \quad t \in[0, T] .
\end{aligned}
$$

For the sake of computational convenience, we introduce

$$
\Lambda_{1}=\frac{T^{3}}{6}\left(1+\left|\lambda_{1} \xi_{1}\right|+3\left|\lambda_{2} \eta_{2}\right| T^{-1}+6\left|\lambda_{3} \eta_{1}\right| T^{-2}\right)
$$

Our first existence result is based on the Leray-Schauder nonlinear alternative.

Theorem 2.4 Assume that $f, g, h, \phi:[0, T] \times \mathbb{R} \rightarrow \mathbb{R}$ are continuous functions and the following conditions hold:

$\left(\mathrm{A}_{1}\right)$ there exist a function $p \in C\left([0, T], \mathbb{R}^{+}\right)$and $\psi: \mathbb{R}^{+} \rightarrow \mathbb{R}^{+}$nondecreasing such that $|f(t, x)| \leq p(t) \psi(\|x\|)$ for each $(t, x) \in[0, T] \times \mathbb{R} ;$

$\left(\mathrm{A}_{2}\right)$ there exist continuous nondecreasing functions $\psi_{j}:[0, \infty) \rightarrow(0, \infty)$ and functions $p_{j} \in$ $C\left([0, T], \mathbb{R}^{+}\right)$such that

$$
|g(t, x)| \leq p_{0}(t) \psi_{0}(\|x\|), \quad|h(t, x)| \leq p_{1}(t) \psi_{1}(\|x\|), \quad|\phi(t, x)| \leq p_{2}(t) \psi_{2}(\|x\|),
$$

for each $(t, x) \in[0, T] \times \mathbb{R}$;

$\left(\mathrm{A}_{3}\right)$ there exists a number $M>0$ such that

$$
\frac{M}{\|p\| \psi(\|x\|) \Lambda_{1}+\left\|p_{0}\right\| \psi_{0}(\|x\|) \Lambda_{2}+\left\|p_{1}\right\| \psi_{1}(\|x\|) \Lambda_{3}+\left\|p_{2}\right\| \psi_{2}(\|x\|) \Lambda_{4}}>1
$$

where $\Lambda_{1}$ is defined by (2.5) and

$$
\Lambda_{2}=\left|\mu_{1} \xi_{1}\right|, \quad \Lambda_{3}=T\left|\mu_{2} \eta_{2}\right|, \quad \Lambda_{4}=T\left|\mu_{3} \eta_{1}\right|
$$

Then the boundary value problem (1.1) has at least one solution on [0,T].

Proof Consider the operator $\mathcal{F}: \mathcal{C} \rightarrow \mathcal{C}$ defined by (2.4). It is easy to prove that $\mathcal{F}$ is continuous. Next, we show that $\mathcal{F}$ maps bounded sets into bounded sets in $C([0, T], \mathbb{R})$. For a positive number $\rho$, let $B_{\rho}=\{x \in C([0, T], \mathbb{R}):\|x\| \leq \rho\}$ be a bounded set in $C([0, T], \mathbb{R})$.

Then, for each $x \in B_{\rho}$, we have

$$
\begin{aligned}
|(\mathcal{F} x)(t)| \leq & \int_{0}^{t} \frac{(t-s)^{2}}{2}|f(s, x(s))| d s+\left|\lambda_{1} \xi_{1}\right| \int_{0}^{T} \frac{(T-s)^{2}}{2}|f(s, x(s))| d s \\
& +\left|\lambda_{2} \eta_{2}\right| \int_{0}^{T}(T-s)|f(s, x(s))| d s+\left|\lambda_{3} \eta_{1}\right| \int_{0}^{T}|f(s, x(s))| d s \\
& +\left|\mu_{1} \xi_{1}\right| \int_{0}^{T}|g(s, x(s))| d s+\left|\mu_{2} \eta_{2}\right| \int_{0}^{T}|h(s, x(s))| d s \\
& +\left|\mu_{3} \eta_{1}\right| \int_{0}^{T}|\phi(s, x(s))| d s \\
\leq & \int_{0}^{t} \frac{(t-s)^{2}}{2} p(s) \psi(\|x\|) d s+\left|\lambda_{1} \xi_{1}\right| \int_{0}^{T} \frac{(T-s)^{2}}{2} p(s) \psi(\|x\|) d s
\end{aligned}
$$




$$
\begin{aligned}
& +\left|\lambda_{2} \eta_{2}\right| \int_{0}^{T}(T-s) p(s) \psi(\|x\|) d s+\left|\lambda_{3} \eta_{1}\right| \int_{0}^{T} p(s) \psi(\|x\|) d s \\
& +\left|\mu_{1} \xi_{1}\right| \int_{0}^{T} p_{0}(s) \psi_{0}(\|x\|) d s+\left|\mu_{2} \eta_{2}\right| \int_{0}^{T} p_{1}(s) \psi_{1}(\|x\|) d s \\
& +\left|\mu_{3} \eta_{1}\right| \int_{0}^{T} p_{2}(s) \psi_{2}(\|x\|) d s \\
& \leq\|p\| \psi(\|x\|) \frac{T^{3}}{6}\left\{1+\left|\lambda_{1} \xi_{1}\right|+3\left|\lambda_{2} \eta_{2}\right| T^{-1}+6\left|\lambda_{3} \eta_{1}\right| T^{-2}\right\} \\
& +T\left\|p_{0}\right\| \psi_{0}(\|x\|)\left|\mu_{1} \xi_{1}\right|+T\left\|p_{1}\right\| \psi_{1}(\|x\|)\left|\mu_{2} \eta_{2}\right|+T\left\|p_{2}\right\| \psi_{2}(\|x\|)\left|\mu_{3} \eta_{1}\right| \\
& =\|p\| \psi(\|x\|) \Lambda_{1}+\left\|p_{0}\right\| \psi_{0}(\|x\|) \Lambda_{2}+\left\|p_{1}\right\| \psi_{1}(\|x\|) \Lambda_{3}+\left\|p_{2}\right\| \psi_{2}(\|x\|) \Lambda_{4} .
\end{aligned}
$$

Thus,

$$
\|\mathcal{F} x\| \leq\|p\| \psi(\rho) \Lambda_{1}+\left\|p_{0}\right\| \psi_{0}(\rho) \Lambda_{2}+\left\|p_{1}\right\| \psi_{1}(\rho) \Lambda_{3}+\left\|p_{2}\right\| \psi_{2}(\rho) \Lambda_{4} .
$$

Now we show that $\mathcal{F}$ maps bounded sets into equicontinuous sets of $C([0, T], \mathbb{R})$. Let $t^{\prime}, t^{\prime \prime} \in[0, T]$ with $t^{\prime}<t^{\prime \prime}$ and $x \in B_{\rho}$, where $B_{\rho}$ is a bounded set of $C([0, T], \mathbb{R})$. Then we have

$$
\begin{aligned}
& \left|(\mathcal{F} x)\left(t^{\prime \prime}\right)-(\mathcal{F} x)\left(t^{\prime}\right)\right| \\
& \leq\left|\psi(\|x\|) \int_{0}^{t^{\prime}}\left[\frac{\left(t^{\prime \prime}-s\right)^{2}-\left(t^{\prime}-s\right)^{2}}{2}\right] p(s) d s+\psi(\|x\|) \int_{t^{\prime}}^{t^{\prime \prime}} \frac{\left(t^{\prime \prime}-s\right)^{2}}{2} p(s) d s\right| \\
& \quad+\left|\left(1-\lambda_{1}\right) \lambda_{2} \xi_{2}\right|\left|t^{\prime \prime}-t^{\prime}\right| \psi(\|x\|) \int_{0}^{T}(T-s) p(s) d s+\left|\lambda_{2} \xi_{3}\right|\left[2\left|\left(1-\lambda_{1}\right) \lambda_{2}\right| T\left|t^{\prime \prime}-t^{\prime}\right|\right. \\
& \left.\quad+\left|\left(1-\lambda_{1}\right)\left(1-\lambda_{2}\right)\right|\left|t^{\prime \prime 2}-t^{\prime 2}\right|\right] \psi(\|x\|) \int_{0}^{T} p(s) d s \\
& \quad+\left|\left(1-\lambda_{1}\right) \mu_{1} \lambda_{2} \xi_{2}\right|\left|t^{\prime \prime}-t^{\prime}\right| \psi_{1}(\|x\|) \int_{0}^{T} p_{1}(s) d s \\
& \quad+\left|\lambda_{3} \xi_{3} \mu_{2}\right|\left[2 T\left|\left(1-\lambda_{1}\right) \lambda_{2}\right|\left|t^{\prime \prime}-t^{\prime}\right|+\left|\left(1-\lambda_{1}\right)\left(1-\lambda_{2}\right)\right|\left|t^{\prime \prime 2}-t^{\prime 2}\right|\right] \\
& \quad \times \psi_{2}(\|x\|) \int_{0}^{T} p_{2}(s) d s .
\end{aligned}
$$

Obviously the right hand side of the above inequality tends to zero independently of $x \in B_{\rho}$ as $t^{\prime \prime}-t^{\prime} \rightarrow 0$. Therefore it follows by the Arzelá-Ascoli theorem that $\mathcal{F} C([0, T], \mathbb{R}) \rightarrow$ $C([0, T], \mathbb{R})$ is completely continuous.

The result will follow from the Leray-Schauder nonlinear alternative (Theorem 2.2) once we have proved the boundedness of the set of all solutions to the equations $x=\lambda \mathcal{F} x$ for $\lambda \in[0,1]$.

Let $x$ be a solution. Then, for $t \in[0, T]$, and using the computations in proving that $\mathcal{F}$ is bounded, we have

$$
\begin{aligned}
|x(t)| \leq & \int_{0}^{t} \frac{(t-s)^{2}}{2} p(s) \psi(\|x\|) d s+\left|\lambda_{1} \xi_{1}\right| \int_{0}^{T} \frac{(T-s)^{2}}{2} p(s) \psi(\|x\|) d s \\
& +\left|\lambda_{2} \eta_{2}\right| \int_{0}^{T}(T-s) p(s) \psi(\|x\|) d s+\left|\lambda_{3} \eta_{1}\right| \int_{0}^{T} p(s) \psi(\|x\|) d s
\end{aligned}
$$




$$
\begin{aligned}
& +\left|\mu_{1} \xi_{1}\right| \int_{0}^{T} p_{0}(s) \psi_{0}(\|x\|) d s+\left|\mu_{2} \eta_{2}\right| \int_{0}^{T} p_{1}(s) \psi_{1}(\|x\|) d s \\
& +\left|\mu_{3} \eta_{1}\right| \int_{0}^{T} p_{2}(s) \psi_{2}(\|x\|) d s \\
\leq & \|p\| \psi(\|x\|) \frac{T^{3}}{6}\left\{1+\left|\lambda_{1} \xi_{1}\right|+3\left|\lambda_{2} \eta_{2}\right| T^{-1}+6\left|\lambda_{3} \eta_{1}\right| T^{-2}\right\} \\
& +T\left\|p_{0}\right\| \psi_{0}(\|x\|)\left|\mu_{1} \xi_{1}\right|+T\left\|p_{1}\right\| \psi_{1}(\|x\|)\left|\mu_{2} \eta_{2}\right|+T\left\|p_{2}\right\| \psi_{2}(\|x\|)\left|\mu_{3} \eta_{1}\right| \\
= & \|p\| \psi(\|x\|) \Lambda_{1}+\left\|p_{0}\right\| \psi_{0}(\|x\|) \Lambda_{2}+\left\|p_{1}\right\| \psi_{1}(\|x\|) \Lambda_{3}+\left\|p_{2}\right\| \psi_{2}(\|x\|) \Lambda_{4} .
\end{aligned}
$$

Consequently, we have

$$
\frac{\|x\|}{\|p\| \psi(\|x\|) \Lambda_{1}+\left\|p_{0}\right\| \psi_{0}(\|x\|) \Lambda_{2}+\left\|p_{1}\right\| \psi_{1}(\|x\|) \Lambda_{3}+\left\|p_{2}\right\| \psi_{2}(\|x\|) \Lambda_{4}} \leq 1
$$

In view of $\left(\mathrm{A}_{3}\right)$, there exists $M$ such that $\|x\| \neq M$. Let us set

$$
U=\{x \in C([0, T], \mathbb{R}):\|x\|<M+1\} .
$$

Note that the operator $\mathcal{F}: \bar{U} \rightarrow C([0, T], \mathbb{R})$ is continuous and completely continuous. From the choice of $U$, there is no $x \in \partial U$ such that $x=\lambda \mathcal{F} x$ for some $\lambda \in(0,1)$. Consequently, by the Leray-Schauder alternative (Theorem 2.2), we deduce that $\mathcal{F}$ has a fixed point $x \in \bar{U}$ which is a solution of the problem (1.1).

Our next result is based on the celebrated fixed point theorem due to Banach.

Theorem 2.5 Assume that $f, g, h, \phi:[0, T] \times \mathbb{R} \rightarrow \mathbb{R}$ are continuous functions satisfying the conditions:

(A $\left.\mathrm{A}_{4}\right)|f(t, x)-f(t, y)| \leq L|x-y|, \forall t \in[0, T], L>0, x, y \in \mathbb{R}$

$\left(\mathrm{A}_{5}\right)|g(t, x)-g(t, y)| \leq \mathcal{L}_{1}|x-y|,|h(t, x)-h(t, y)| \leq \mathcal{L}_{2}|x-y|,|\phi(t, x)-\phi(t, y)| \leq \mathcal{L}_{3}|x-y|$, $\forall t \in[0, T], \mathcal{L}_{j}>0, j=1,2,3, x, y \in \mathbb{R}$.

Then the boundary value problem (1.1) has a unique solution if

$$
L \Lambda_{1}+\left\{\mathcal{L}_{0}\left|\mu_{1} \xi_{1}\right|+\mathcal{L}_{1}\left|\mu_{2} \eta_{2}\right|+\mathcal{L}_{2}\left|\mu_{3} \eta_{1}\right|\right\} T<1
$$

where $\Lambda_{1}$ is given by (2.5).

Proof Let us fix $\sup _{t \in[0, T]}|f(t, 0)|=M, \sup _{t \in[0, T]}|g(t, 0)|=\mathcal{M}_{0}, \sup _{t \in[0, T]}|h(t, 0)|=\mathcal{M}_{1}$, $\sup _{t \in[0, T]}|\phi(t, 0)|=\mathcal{M}_{2}$ and choose

$$
r \geq \frac{M \Lambda_{1}+\left\{\mathcal{M}_{0}\left|\mu_{1} \xi_{1}\right|+\mathcal{M}_{1}\left|\mu_{2} \eta_{2}\right|+\mathcal{M}_{2}\left|\mu_{3} \eta_{1}\right|\right\} T}{1-\left(L \Lambda_{1}+\left\{\mathcal{L}_{0}\left|\mu_{1} \xi_{1}\right|+\mathcal{L}_{1}\left|\mu_{2} \eta_{2}\right|+\mathcal{L}_{2}\left|\mu_{3} \eta_{1}\right|\right\} T\right)}
$$


Then we show that $\mathcal{F} B_{r} \subset B_{r}$, where $B_{r}=\{x \in \mathcal{C}:\|x\| \leq r\}$. For $x \in B_{r}$, we have

$$
\begin{aligned}
|(\mathcal{F} x)(t)| \leq & \sup _{t \in[0, T]}\left\{\int_{0}^{t} \frac{(t-s)^{2}}{2}|f(s, x(s))| d s+\left|\lambda_{1} \xi_{1}\right| \int_{0}^{T} \frac{(T-s)^{2}}{2}|f(s, x(s))| d s\right. \\
& +\left|\lambda_{2} \eta_{2}\right| \int_{0}^{T}(T-s)|f(s, x(s))| d s+\left|\lambda_{3} \eta_{1}\right| \int_{0}^{T}|f(s, x(s))| d s \\
& +\left|\mu_{1} \xi_{1}\right| \int_{0}^{T}\left|g_{0}(s, x(s))\right| d s+\left|\mu_{2} \eta_{2}\right| \int_{0}^{T}\left|g_{1}(s, x(s))\right| d s \\
& \left.+\left|\mu_{3} \eta_{1}\right| \int_{0}^{T}\left|g_{2}(s, x(s))\right| d s\right\} \\
\leq & \sup _{t \in[0, T]}\left\{\int_{0}^{t} \frac{(t-s)^{2}}{2}[|f(s, x(s))-f(s, 0)|+|f(s, 0)|] d s\right. \\
& +\left|\lambda_{1} \xi_{1}\right| \int_{0}^{T} \frac{(T-s)^{2}}{2}[|f(s, x(s))-f(s, 0)|+|f(s, 0) d s|] d s \\
& +\left|\lambda_{2} \eta_{2}\right| \int_{0}^{T}(T-s)[|f(s, x(s))-f(s, 0)|+|f(s, 0) d s|] d s \\
& +\left|\lambda_{3} \eta_{1}\right| \int_{0}^{T}[|f(s, x(s))-f(s, 0)|+|f(s, 0)|] d s \\
& +\left|\mu_{1} \xi_{1}\right| \int_{0}^{T}[|g(s, x(s))-g(s, 0)|+|g(s, 0)|] d s \\
& +\left|\mu_{2} \eta_{2}\right| \int_{0}^{T}[|h(s, x(s))-h(s, 0)|+|h(s, 0)|] d s \\
& \left.+\left|\mu_{3} \eta_{1}\right| \int_{0}^{T}[|\phi 2(s, x(s))-\phi(s, 0)|+|\phi(s, 0)|] d s\right\} \\
\leq & \left(L r+\Lambda_{1}+\mathcal{L}_{0} r+\mathcal{L}_{0} \mid \mu_{1} \mathcal{M}_{0}\right)\left|\mu_{1} \xi_{1}\right| T+\xi_{1} \mid T+\left(\mathcal{L}_{1} r+\mathcal{L}_{1}\left|\mu_{2} \eta_{2}\right| T+\mathcal{L}_{2}\left|\mu_{2}\right| \mu_{3} \eta_{1} \mid T\right) r \\
& +\left(M \Lambda_{1}+\mathcal{M}_{0}\left|\mu_{1} \xi_{1}\right| T+\mathcal{M}_{1}\left|\mu_{1} \eta_{2}\right| T+\mathcal{L}_{2} r+\mathcal{M}_{2}\left|\mu_{2} \eta_{1}\right| T\right)\left|\mu_{3} \eta_{1}\right| T \\
& \\
&
\end{aligned}
$$

Now, for $x, y \in \mathcal{C}$ and for each $t \in[0, T]$, we obtain

$$
\begin{aligned}
|(\mathcal{F} x)(t)-(\mathcal{F} y)(t)| \leq & \sup _{t \in[0, T]}\left\{\int_{0}^{t} \frac{(t-s)^{2}}{2}|f(s, x(s))-f(s, y(s))| d s\right. \\
& +\left|\lambda_{1} \xi_{1}\right| \int_{0}^{T} \frac{(T-s)^{2}}{2}|f(s, x(s))-f(s, y(s))| d s \\
& +\left|\lambda_{2} \eta_{2}\right| \int_{0}^{T}(T-s)|f(s, x(s))-f(s, y(s))| d s \\
& +\left|\lambda_{3} \eta_{1}\right| \int_{0}^{T}|f(s, x(s))-f(s, y(s))| d s \\
& +\left|\mu_{1} \xi_{1}\right| \int_{0}^{T}|g(s, x(s))-g(s, y(s))| d s
\end{aligned}
$$




$$
\begin{aligned}
& +\left|\mu_{2} \eta_{2}\right| \int_{0}^{T}|h(s, x(s))-h(s, y(s))| d s \\
& \left.+\left|\mu_{3} \eta_{1}\right| \int_{0}^{T}|\phi(s, x(s))-\phi(s, y(s))| d s\right\} \\
\leq & \|x-y\| \frac{L T^{3}}{6}\left\{1+\left|\lambda_{1} \xi_{1}\right|+3\left|\lambda_{2} \eta_{2}\right| T^{-1}+6\left|\lambda_{3} \eta_{1}\right| T^{-2}\right\} \\
& +\mathcal{L}_{0}\|x-y\|\left|\mu_{1} \xi_{1}\right|+\mathcal{L}_{1}\|x-y\|\left|\mu_{2} \eta_{2}\right| T+\mathcal{L}_{2}\left|\mu_{3} \eta_{1}\right| T\|x-y\| \\
= & \left(L \Lambda_{1}+\mathcal{L}_{0}\left|\mu_{1} \xi_{1}\right| T+\mathcal{L}_{1}\left|\mu_{2} \eta_{2}\right| T+\mathcal{L}_{2}\left|\mu_{3} \eta_{1}\right| T\right)\|x-y\|,
\end{aligned}
$$

which implies that $\|\mathcal{F} x-\mathcal{F} y\| \leq\left(L \Lambda_{1}+\left(\mathcal{L}_{0}\left|\mu_{1} \xi_{1}\right|+\mathcal{L}_{1}\left|\mu_{2} \eta_{2}\right|+\mathcal{L}_{2}\left|\mu_{3} \eta_{1}\right|\right) T\right)\|x-y\|$. As $L \Lambda_{1}+\left(\mathcal{L}_{0}\left|\mu_{1} \xi_{1}\right|+\mathcal{L}_{1}\left|\mu_{2} \eta_{2}\right|+\mathcal{L}_{2}\left|\mu_{3} \eta_{1}\right|\right) T<1, \mathcal{F}$ is a contraction. Thus, the conclusion of the theorem follows by the contraction mapping principle (Banach fixed point theorem).

Theorem 2.6 Let $f, g, h, \phi:[0, T] \times \mathbb{R} \rightarrow \mathbb{R}$ be continuous functions satisfying the assumptions $\left(\mathrm{A}_{4}\right)-\left(\mathrm{A}_{5}\right)$. In addition we suppose that

( $\left.\mathrm{A}_{6}\right)|f(t, x)| \leq v(t), \forall(t, x) \in[0, T] \times \mathbb{R}$, and $v \in C\left([0, T], \mathbb{R}^{+}\right)$;

$\left(\mathrm{A}_{7}\right)|g(t, x)| \leq v_{0}(t),|h(t, x)| \leq v_{1}(t),|\phi(t, x)| \leq v_{2}(t), \forall(t, x) \in[0, T] \times \mathbb{R}$, and $v_{j} \in$ $C\left([0, T], \mathbb{R}^{+}\right), j=0,1,2$.

If

$$
\frac{L\left(6 \Lambda_{1}-T^{3}\right)}{6}+\left(\mathcal{L}_{0}\left|\mu_{1} \xi_{1}\right|+\mathcal{L}_{1}\left|\mu_{2} \eta_{2}\right|+\mathcal{L}_{2}\left|\mu_{3} \eta_{1}\right|\right) T<1,
$$

then problem (1.1) has at least one solution on $[0, T]$.

Proof Letting $\sup _{t \in[0, T]}|v(t)|=\|v\|, \sup _{t \in[0, T]}\left|v_{j}(t)\right|=\left\|v_{j}\right\|, j=0,1,2$, we fix

$$
\bar{r} \geq \Lambda_{1}\|v\|+\left(\left|\mu_{1} \xi_{1}\right|\left\|v_{0}\right\|+\left|\mu_{2} \eta_{2}\right|\left\|v_{1}\right\|+\left|\mu_{3} \eta_{1}\right|\left\|v_{2}\right\|\right) T
$$

and consider $B_{\bar{r}}=\{x \in \mathcal{C}:\|x\| \leq \bar{r}\}$. We define the operators $\mathcal{P}$ and $\mathcal{Q}$ on $B_{\bar{r}}$ as

$$
\begin{aligned}
(\mathcal{P} x)(t)= & \int_{0}^{t} \frac{(t-s)^{2}}{2} f(s, x(s)) d s, \\
(\mathcal{Q} x)(t)= & -\lambda_{1} \xi_{1} \int_{0}^{T} \frac{(T-s)^{2}}{2} f(s, x(s)) d s+\lambda_{2} \eta_{2} \int_{0}^{T}(T-s) f(s, x(s)) d s \\
& +\lambda_{3} \eta_{1} \int_{0}^{T} f(s, x(s)) d s-\mu_{1} \xi_{1} \int_{0}^{T} g(s, x(s)) d s \\
& +\mu_{2} \eta_{2} \int_{0}^{T} h(s, x(s)) d s+\mu_{3} \eta_{1} \int_{0}^{T} \phi(s, x(s)) d s, \quad t \in[0, T] .
\end{aligned}
$$

For $x, y \in B_{\bar{r}}$, we find that

$$
\|\mathcal{P} x+\mathcal{Q} y\| \leq \Lambda_{1}\|v\|+\left(\left|\mu_{1} \xi_{1}\right|\left\|\nu_{0}\right\|+\left|\mu_{2} \eta_{2}\right|\left\|v_{1}\right\|+\left|\mu_{3} \eta_{1}\right|\left\|\nu_{2}\right\|\right) T \leq \bar{r} .
$$

Thus, $\mathcal{P} x+\mathcal{Q} y \in B_{\bar{r}}$. It follows from the assumption $\left(\mathrm{A}_{4}\right)$ together with (2.6) that $\mathcal{Q}$ is a contraction mapping. Continuity of $f$ implies that the operator $\mathcal{P}$ is continuous. Also, $\mathcal{P}$ 
is uniformly bounded on $B_{\bar{r}}$ as

$$
\|\mathcal{P} x\| \leq \frac{T^{3}}{6}\|\mu\|
$$

Now we prove the compactness of the operator $\mathcal{P}$.

We define $\sup _{(t, x) \in[0, T] \times B_{\bar{r}}}|f(t, x)|=f_{s}<\infty$, and consequently, for $t_{1}, t_{2} \in[0, T]$ with $t_{1}<t_{2}$, we have

$$
\left|(\mathcal{P} x)\left(t_{2}\right)-(\mathcal{P} x)\left(t_{1}\right)\right| \leq \frac{f_{s}}{\Gamma(q)}\left|\int_{0}^{t_{1}}\left[\left(t_{2}-s\right)^{2}-\left(t_{1}-s\right)^{2}\right] d s+\int_{t_{1}}^{t_{2}}\left(t_{2}-s\right)^{2} d s\right|
$$

which is independent of $x$. Thus, $\mathcal{P}$ is equicontinuous. So $\mathcal{P}$ is relatively compact on $B_{\bar{r}}$. Hence, by the Arzelá-Ascoli theorem, $\mathcal{P}$ is compact on $B_{\bar{r}}$. Thus all the assumptions of Lemma 2.3 are satisfied. So the conclusion of Lemma 2.3 implies that the boundary value problem (1.1) has at least one solution on $[0, T]$.

Example 2.7 Consider the following boundary value problem:

$$
\left\{\begin{array}{l}
x^{\prime \prime \prime}(t)=\frac{1}{8}\left(\cos t+\tan ^{-1} x(t)\right), \quad t \in[0,1] \\
x(0)+x(1)=\int_{0}^{1} \frac{x(s)}{(1+s)^{2}} d s \\
x^{\prime}(0)+x^{\prime}(1)=\frac{1}{2} \int_{0}^{1}\left(\frac{e^{s} x(s)}{1+2 e^{s}}+\frac{1}{2}\right) d s \\
x^{\prime \prime}(0)+x^{\prime \prime}(1)=\frac{1}{3} \int_{0}^{1}\left(\frac{x(s)}{1+e^{s}}+\frac{3}{4}\right) d s,
\end{array}\right.
$$

where

$$
\begin{aligned}
& f(t, x)=\frac{1}{8}\left(\cos t+\tan ^{-1} x(t)\right), \quad g(t, x)=\frac{x(t)}{(1+t)^{2}}, \\
& h(t, x)=\frac{e^{t} x(t)}{1+2 e^{t}}+\frac{1}{2}, \quad \phi(t, x)=\frac{x(t)}{1+e^{t}}+\frac{3}{4},
\end{aligned}
$$

and $\lambda_{1}=\lambda_{2}=\lambda_{3}=-1, \mu_{1}=1, \mu_{2}=\frac{1}{2}, \mu_{3}=\frac{1}{3}$.

Clearly, $\xi_{1}=-1 / 2, \xi_{2}=1 / 4, \xi_{3}=-1 / 16, \eta_{1}=1 / 16, \eta_{2}=1 / 4$

$$
\begin{aligned}
& |f(t, x)-f(t, y)| \leq L|x-y|, \quad|g(t, x)-g(t, y)| \leq|x-y|, \\
& |h(t, x)-h(t, y)| \leq \frac{1}{3}|x-y|, \quad|\phi(t, x)-\phi(t, y)| \leq \frac{1}{2}|x-y|, \\
& \mathcal{L}_{0}=1, \quad \mathcal{L}_{1}=\frac{1}{3}, \quad \mathcal{L}_{2}=\frac{1}{2}, \\
& \Lambda_{1}=\frac{T^{3}}{6}\left(1+\left|\lambda_{1} \xi_{1}\right|+3\left|\lambda_{2} \eta_{2}\right| T^{-1}+6\left|\lambda_{3} \eta_{1}\right| T^{-2}\right) \approx 0.4375,
\end{aligned}
$$

and

$$
L \Lambda_{1}-\frac{T^{3}}{6}+\left\{\mathcal{L}_{0}\left|\mu_{1} \xi_{1}\right|+\mathcal{L}_{1}\left|\mu_{2} \eta_{2}\right|+\mathcal{L}_{2}\left|\mu_{3} \eta_{1}\right|\right\} T \approx 0.4401041<1 .
$$

Thus, all the conditions of Theorem 2.5 are satisfied. So there exists at least one solution of the problem $(2.7)$ on $[0,1]$. 


\section{Problem II}

We need to establish the following result to define the solutions for the given problem.

Lemma 3.1 For any $\sigma \in C([0,1], \mathbb{R})$, the unique solution of the boundary value problem

$$
\left\{\begin{array}{l}
x^{\prime \prime \prime}(t)=\sigma(t), \quad 0<t<1, \\
x(0)=0, \quad x^{\prime}(0)=0, \\
x(1)=\sum_{i=1}^{n-2} \alpha_{i} \int_{\zeta_{i}}^{\eta_{i}} x(s) d s, \quad 0<\zeta_{i}<\eta_{i}<1, i=1,2, \ldots, n-2, n \geq 3
\end{array}\right.
$$

is given by

$$
\begin{aligned}
x(t)= & \int_{0}^{t} \frac{(t-s)^{2}}{2} \sigma(s) d s-\frac{3 t^{2}}{\left(3-\sum_{i=1}^{n-2} \alpha_{i}\left(\eta_{i}^{3}-\zeta_{i}^{3}\right)\right)}\left[\int_{0}^{1} \frac{(1-s)^{2}}{2} \sigma(s) d s\right. \\
& \left.-\sum_{i=1}^{n-2} \alpha_{i} \int_{\zeta_{i}}^{\eta_{i}}\left(\int_{0}^{s} \frac{(s-u)^{2}}{2} \sigma(u) d u\right) d s\right] .
\end{aligned}
$$

Proof The general solution of the fractional differential equation in (3.1) can be written as

$$
x(t)=\int_{0}^{t} \frac{(t-s)^{2}}{2 !} \sigma(s) d s-c_{0}-c_{1} t-c_{2} t^{2},
$$

where $c_{0}, c_{1}, c_{2}$ are arbitrary constants. Applying the boundary conditions for the problem (3.1), we find that $c_{0}=0, c_{1}=0$, and

$$
\begin{aligned}
c_{2}= & \frac{3}{\left(3-\sum_{i=1}^{n-2} \alpha_{i}\left(\eta_{i}^{3}-\zeta_{i}^{3}\right)\right)}\left[\int_{0}^{1} \frac{(1-s)^{2}}{2} \sigma(s) d s\right. \\
& \left.-\sum_{i=1}^{n-2} \alpha_{i} \int_{\zeta_{i}}^{\eta_{i}}\left(\int_{0}^{s} \frac{(s-u)^{2}}{2} \sigma(u) d u\right) d s\right] .
\end{aligned}
$$

Substituting the values of $c_{0}, c_{1}, c_{2}$ in (3.3) yields the solution (3.2).

\subsection{Existence of solutions}

In view of Lemma 3.1, we transform problem (1.2) as

$$
x=S x .
$$

In (3.4), the operator $S: \mathcal{C} \rightarrow \mathcal{C}$ is defined by

$$
\begin{aligned}
(S x)(t)= & \int_{0}^{t} \frac{(t-s)^{2}}{2} f(s, x(s)) d s-\vartheta t^{2}\left[\int_{0}^{1} \frac{(1-s)^{2}}{2} f(s, x(s)) d s\right. \\
& \left.-\sum_{i=1}^{n-2} \alpha_{i} \int_{\zeta_{i}}^{\eta_{i}}\left(\int_{0}^{s} \frac{(s-u)^{2}}{2} f(u, x(u)) d u\right) d s\right], \quad t \in[0,1],
\end{aligned}
$$

where

$$
\vartheta=3\left(3-\sum_{i=1}^{n-2} \alpha_{i}\left(\eta_{i}^{3}-\zeta_{i}^{3}\right)\right)^{-1}
$$


For convenience, let us set

$$
\Lambda=\frac{1}{6}\left[1+|\vartheta|\left\{1+\sum_{i=1}^{n-2} \frac{\alpha_{i}\left(\eta_{i}^{4}-\zeta_{i}^{4}\right)}{4}\right\}\right] .
$$

Theorem 3.2 Assume that $f:[0,1] \times \mathbb{R} \rightarrow \mathbb{R}$ is a jointly continuous function and satisfies the assumption

( $\left.\mathrm{A}_{1}\right)|f(t, x)-f(t, y)| \leq L|x-y|, \forall t \in[0,1], L>0, x, y \in \mathbb{R}$,

with $L<1 / \Lambda$, where $\Lambda$ is given by (3.6). Then the boundary value problem (1.2) has a unique solution.

Proof Setting $\sup _{t \in[0,1]}|f(t, 0)|=M$ and choosing $r \geq \frac{\Lambda M}{1-L \Lambda}$, we show that $S B_{r} \subset B_{r}$, where $B_{r}=\{x \in \mathcal{C}:\|x\| \leq r\}$. For $x \in B_{r}$, we have

$$
\begin{aligned}
|(S x)(t)| \leq & \sup _{t \in[0,1]}\left\{\int_{0}^{t} \frac{(t-s)^{2}}{2}|f(s, x(s))| d s+|\vartheta| t^{2}\left[\int_{0}^{1} \frac{(1-s)^{2}}{2}|f(s, x(s))| d s\right.\right. \\
& \left.\left.+\sum_{i=1}^{n-2} \alpha_{i} \int_{\zeta_{i}}^{\eta_{i}}\left(\int_{0}^{s} \frac{(s-u)^{2}}{2}|f(u, x(u))| d u\right) d s\right]\right\} \\
\leq & \sup _{t \in[0,1]}\left\{\frac{1}{2} \int_{0}^{t}(t-s)^{2}(|f(s, x(s))-f(s, 0)|+|f(s, 0)|) d s\right. \\
& +|\vartheta| t^{2}\left[\int_{0}^{1} \frac{(1-s)^{2}}{2}(|f(s, x(s))-f(s, 0)|+|f(s, 0)|) d s\right. \\
& \left.\left.+\sum_{i=1}^{n-2} \alpha_{i} \int_{\zeta_{i}}^{\eta_{i}}\left(\int_{0}^{s} \frac{(s-u)^{2}}{2}(|f(u, x(u))-f(u, 0)|+|f(u, 0)|) d u\right) d s\right]\right\} \\
\leq & (L r+M) \sup _{t \in[0,1]}\left\{\frac{1}{2} \int_{0}^{t}(t-s)^{2} d s\right. \\
& \left.+|\vartheta| t^{2}\left[\int_{0}^{1} \frac{(1-s)^{2}}{2} d s+\sum_{i=1}^{n-2} \alpha_{i} \int_{\zeta_{i}}^{\eta_{i}}\left(\int_{0}^{s} \frac{(s-u)^{2}}{2} d u\right) d s\right]\right\} \\
\leq & \frac{(L r+M)\left[1+|\vartheta|\left\{1+\sum_{i=1}^{n-2} \frac{\alpha_{i}\left(\eta_{i}^{4}-\zeta_{i}^{4}\right)}{4}\right\}\right]}{6}=(L r+M) \Lambda \leq r .
\end{aligned}
$$

Now, for $x, y \in \mathcal{C}$ and for each $t \in[0,1]$, we obtain

$$
\begin{aligned}
& |(S x)(t)-(S y)(t)| \\
& \leq \sup _{t \in[0,1]}\left\{\int_{0}^{t} \frac{(t-s)^{2}}{2}|f(s, x(s))-f(s, y(s))| d s\right. \\
& \quad+|\vartheta| t^{2}\left[\int_{0}^{1} \frac{(1-s)^{2}}{2}|f(s, x(s))-f(s, y(s))| d s\right.
\end{aligned}
$$




$$
\begin{aligned}
& \left.\left.+\sum_{i=1}^{n-2} \alpha_{i} \int_{\zeta_{i}}^{\eta_{i}}\left(\int_{0}^{s} \frac{(s-u)^{2}}{2}|f(u, x(u))-f(u, y(u))| d u\right) d s\right]\right\} \\
\leq & L\|x-y\| \sup _{t \in[0,1]}\left\{\frac{1}{2} \int_{0}^{t}(t-s)^{2} d s\right. \\
& \left.+|\vartheta| t^{2}\left[\int_{0}^{1} \frac{(1-s)^{2}}{2} d s+\sum_{i=1}^{n-2} \alpha_{i} \int_{\zeta_{i}}^{\eta_{i}}\left(\int_{0}^{s} \frac{(s-u)^{2}}{2} d u\right) d s\right]\right\} \\
\leq & \frac{L}{6}\left[1+|\vartheta|\left\{1+\sum_{i=1}^{n-2} \frac{\alpha_{i}\left(\eta_{i}^{4}-\zeta_{i}^{4}\right)}{4}\right\}\right]\|x-y\|=L \Lambda\|x-y\|,
\end{aligned}
$$

where $\Lambda$ is given by (3.6). Observe that $\Lambda$ depends only on the parameters involved in the problem. Hence $\|S x-S y\| \leq L \Lambda\|x-y\|$ and as $L<1 / \Lambda$, therefore $S$ is a contraction. Thus, the conclusion of the theorem follows by the contraction mapping principle (Banach fixed point theorem).

Now, we prove the existence of solutions of the problem (1.2) by applying Krasnoselskii's fixed point theorem.

Theorem 3.3 Let $f:[0,1] \times \mathbb{R} \rightarrow \mathbb{R}$ be a jointly continuous function satisfying the assumption $\left(\mathrm{A}_{1}\right)$. Moreover, we assume that

$\left(\mathrm{A}_{2}\right)|f(t, x)| \leq \mu(t), \forall(t, x) \in[0,1] \times \mathbb{R}$, and $\mu \in C\left([0,1], \mathbb{R}^{+}\right)$.

If

$$
\frac{L|\vartheta|}{6}\left(1+\sum_{i=1}^{n-2} \frac{\alpha_{i}\left(\eta_{i}^{4}-\zeta_{i}^{4}\right)}{4}\right)<1
$$

then the boundary value problem (1.2) has at least one solution on $[0,1]$.

Proof By the assumption $\left(\mathrm{A}_{2}\right)$, we can fix

$$
\bar{r} \geq \frac{\|\mu\|}{6}\left[1+|\vartheta|\left(1+\sum_{i=1}^{n-2} \frac{\alpha_{i}\left(\eta_{i}^{4}-\zeta_{i}^{4}\right)}{4}\right)\right],
$$

and consider $B_{\bar{r}}=\{x \in \mathcal{C}:\|x\| \leq \bar{r}\}$. For $t \in[0,1]$, we define the operators $\mathcal{P}_{S}$ and $\mathcal{Q}_{S}$ on $B_{\bar{r}}$ as

$$
\begin{aligned}
& \left(\mathcal{P}_{S} x\right)(t)=\int_{0}^{t} \frac{(t-s)^{2}}{2} f(s, u(s)) d s \\
& \left(\mathcal{Q}_{S} x\right)(t)=-\vartheta t^{2}\left[\int_{0}^{1} \frac{(1-s)^{2}}{2} f(s, x(s)) d s-\sum_{i=1}^{n-2} \alpha_{i} \int_{\zeta_{i}}^{\eta_{i}}\left(\int_{0}^{s} \frac{(s-u)^{2}}{2} f(u, x(u)) d u\right) d s\right] .
\end{aligned}
$$

For $x, y \in B_{\bar{r}}$, we find that

$$
\left\|\mathcal{P}_{S} x+\mathcal{Q}_{S} y\right\| \leq \frac{\|\mu\|}{6}\left[1+|\vartheta|\left(1+\sum_{i=1}^{n-2} \frac{\alpha_{i}\left(\eta_{i}^{4}-\zeta_{i}^{4}\right)}{4}\right)\right] \leq \bar{r} .
$$


Thus, $\mathcal{P}_{S} x+\mathcal{Q}_{S} y \in B_{\bar{r}}$. It follows from the assumption $\left(\mathrm{A}_{1}\right)$ together with (3.7) that $\mathcal{Q}_{S}$ is a contraction mapping. Continuity of $f$ implies that the operator $\mathcal{P}_{S}$ is continuous. Also, $\mathcal{P}_{S}$ is uniformly bounded on $B_{\bar{r}}$ as

$$
\left\|\mathcal{P}_{S} x\right\| \leq \frac{\|\mu\|}{6} .
$$

Now we prove the compactness of the operator $\mathcal{P}_{S}$. In view of $\left(\mathrm{A}_{1}\right)$, we define

$$
\sup _{(t, x) \in[0,1] \times B_{\bar{r}}}|f(t, x)|=\bar{f} .
$$

Consequently we have

$$
\begin{aligned}
\left|\left(\mathcal{P}_{S} x\right)\left(t_{1}\right)-\left(\mathcal{P}_{S} x\right)\left(t_{2}\right)\right|= & \mid \frac{1}{2} \int_{0}^{t_{1}}\left[\left(t_{2}-s\right)^{2}-\left(t_{1}-s\right)^{2}\right] f(s, x(s)) d s \\
& +\int_{t_{1}}^{t_{2}}\left(t_{2}-s\right)^{2} f(s, x(s)) d s \mid \\
\leq & \frac{\bar{f}}{6}\left(2\left|t_{2}-t_{1}\right|^{3}+\left|t_{2}^{3}-t_{2}^{3}\right|\right),
\end{aligned}
$$

which is independent of $x$ and tends to zero as $t_{2} \rightarrow t_{1}$. Thus, $\mathcal{P}_{S}$ is relatively compact on $B_{\bar{r}}$. Hence, by the Arzelá-Ascoli theorem, $\mathcal{P}_{S}$ is compact on $B_{\bar{r}}$. Thus all the assumptions of Lemma 2.3 are satisfied. So by the conclusion of Lemma 2.3, problem (1.2) has at least one solution on $[0,1]$.

Our next result relies on the Leray-Schauder alternative.

Theorem 3.4 Let $f:[0,1] \times \mathbb{R} \rightarrow \mathbb{R}$ be a jointly continuous function. Assume that:

$\left(\mathrm{A}_{3}\right)$ There exist a function $p \in C\left([0,1], \mathbb{R}^{+}\right)$and a nondecreasing function $\psi: \mathbb{R}^{+} \rightarrow \mathbb{R}^{+}$ such that $|f(t, x)| \leq p(t) \psi(\|x\|), \forall(t, x) \in[0,1] \times \mathbb{R}$.

$\left(\mathrm{A}_{4}\right)$ There exists a constant $M>0$ such that

$$
\frac{M}{\frac{\|p\| \psi(M)}{6}\left[1+|\vartheta|\left\{1+\sum_{i=1}^{n-2} \alpha_{i} \frac{\eta_{i}^{4}-\zeta_{i}^{4}}{4}\right\}\right]}>1 .
$$

Then the boundary value problem (1.2) has at least one solution on $[0,1]$.

Proof Consider the operator $S: \mathcal{C} \rightarrow \mathcal{C}$ with $x=S(x)$, where $S$ is defined by (3.5). We show that $S$ maps bounded sets into bounded sets in $C([0,1], \mathbb{R})$. For a positive number $r$, let $B_{r}=\{x \in C([0,1], \mathbb{R}):\|x\| \leq r\}$ be a bounded set in $C([0,1], \mathbb{R})$. Then

$$
\begin{aligned}
|(S x)(t)| \leq & \int_{0}^{t} \frac{(t-s)^{2}}{2}|f(s, x(s))| d s+|\vartheta| t^{2}\left[\int_{0}^{1} \frac{(1-s)^{2}}{2}|f(s, x(s))| d s\right. \\
& \left.+\sum_{i=1}^{n-2} \alpha_{i} \int_{\zeta_{i}}^{\eta_{i}}\left(\int_{0}^{s} \frac{(s-u)^{2}}{2}|f(u, x(u))| d u\right) d s\right] \\
\leq & \int_{0}^{t} \frac{(t-s)^{2}}{2} p(s) \psi(\|x\|) d s+|\vartheta| t^{2} \int_{0}^{1} \frac{(1-s)^{2}}{2} p(s) \psi(\|x\|) d s
\end{aligned}
$$




$$
\begin{aligned}
& +|\vartheta| t^{2} \sum_{i=1}^{n-2} \alpha_{i} \int_{\zeta_{i}}^{\eta_{i}}\left(\int_{0}^{s} \frac{(s-u)^{2}}{2} p(s) \psi(\|x\|) d u\right) d s \\
\leq & \frac{\|p\| \psi(\|x\|)}{2} \int_{0}^{1}(1-s)^{2} d s+\frac{|\vartheta|\|p\| \psi(\|x\|)}{2} \int_{0}^{1}(1-s)^{2} d s \\
& +\frac{|\vartheta|\|p\| \psi(\|x\|)}{2} \sum_{i=1}^{n-2} \alpha_{i} \int_{\zeta_{i}}^{\eta_{i}}\left(\int_{0}^{s}(s-u)^{2} d u\right) d s \\
\leq & \frac{\|p\| \psi(\|x\|)}{6}+\frac{|\vartheta|\|p\| \psi(\|x\|)}{6}+\frac{|\vartheta|\|p\| \psi(\|x\|)}{2} \sum_{i=1}^{n-2} \alpha_{i} \frac{\eta_{i}^{4}-\zeta_{i}^{4}}{4} \\
= & \frac{\|p\| \psi(\|x\|)}{6}\left[1+|\vartheta|\left\{1+\sum_{i=1}^{n-2} \alpha_{i} \frac{\eta_{i}^{4}-\zeta_{i}^{4}}{4}\right\}\right] .
\end{aligned}
$$

Next we show that $S$ maps bounded sets into equicontinuous sets of $C([0,1], \mathbb{R})$. Let $t^{\prime}, t^{\prime \prime} \in$ $[0,1]$ with $t^{\prime}<t^{\prime \prime}$ and $x \in B_{r}$, where $B_{r}$ is a bounded set of $C([0,1], \mathbb{R})$. Then we obtain

$$
\begin{aligned}
\left|(S x)\left(t^{\prime \prime}\right)-(S x)\left(t^{\prime}\right)\right|= & \mid \frac{1}{2} \int_{0}^{t^{\prime \prime}}\left(t^{\prime \prime}-s\right)^{2} f(s, x(s)) d s-\frac{1}{2} \int_{0}^{t^{\prime}}\left(t^{\prime}-s\right)^{2} f(s, x(s)) d s \\
& -\vartheta\left(\left(t^{\prime \prime}\right)^{2}-\left(t^{\prime}\right)^{2}\right)\left[\int_{0}^{1} \frac{(1-s)^{2}}{2} f(s, x(s)) d s\right. \\
& \left.-\sum_{i=1}^{n-2} \alpha_{i} \int_{\zeta_{i}}^{\eta_{i}}\left(\int_{0}^{s} \frac{(s-u)^{2}}{2} f(u, x(u)) d u\right) d s\right] \mid \\
\leq & \left|\frac{1}{2} \int_{0}^{t^{\prime}}\left[\left(t^{\prime \prime}-s\right)^{2}-\left(t^{\prime}-s\right)^{2}\right] \psi(r) p(s) d s\right| \\
& +\left|\frac{1}{2} \int_{t^{\prime}}^{t^{\prime \prime}}\left(t^{\prime \prime}-s\right)^{2} \psi(r) p(s) d s\right| \\
& +\mid \vartheta\left(\left(t^{\prime \prime}\right)^{2}-\left(t^{\prime}\right)^{2}\right)\left[\int_{0}^{1} \frac{(1-s)^{2}}{2} \psi(r) p(s) d s\right. \\
& \left.+\sum_{i=1}^{n-2} \alpha_{i} \int_{\zeta_{i}}^{\eta_{i}}\left(\int_{0}^{s} \frac{(s-u)^{2}}{2} \psi(r) p(u) d u\right) d s\right] .
\end{aligned}
$$

Obviously the right hand side of the above inequality tends to zero independently of $x \in B_{r}$ as $t^{\prime \prime}-t^{\prime} \rightarrow 0$. As $S$ satisfies the above assumptions, it follows by the Arzelá-Ascoli theorem that $S: C([0,1], \mathbb{R}) \rightarrow C([0,1], \mathbb{R})$ is completely continuous.

The result will follow from the Leray-Schauder nonlinear alternative (Lemma 2.2) once we have proved the boundedness of the set of all solutions to the equations $x=\lambda S x$ for $\lambda \in[0,1]$.

Let $x$ be a solution. Then, for $t \in[0,1]$, and using the computations in proving that $S$ is bounded, we have

$$
\begin{aligned}
|x(t)| & =|\lambda(S x)(t)| \\
& \leq \int_{0}^{t} \frac{(t-s)^{2}}{2}|f(s, x(s))| d s+|\vartheta| t^{2}\left[\int_{0}^{1} \frac{(1-s)^{2}}{2}|f(s, x(s))| d s\right.
\end{aligned}
$$




$$
\begin{aligned}
& \left.+\sum_{i=1}^{n-2} \alpha_{i} \int_{\zeta_{i}}^{\eta_{i}}\left(\int_{0}^{s} \frac{(s-u)^{2}}{2}|f(u, x(u))| d u\right) d s\right] \\
\leq & \frac{\|p\| \psi(\|x\|)}{6}\left[1+|\vartheta|\left\{1+\sum_{i=1}^{n-2} \alpha_{i} \frac{\eta_{i}^{4}-\zeta_{i}^{4}}{4}\right\}\right] .
\end{aligned}
$$

Consequently, we have

$$
\frac{\|x\|}{\frac{\|p\| \psi(\|x\|)}{6}\left[1+|\vartheta|\left\{1+\sum_{i=1}^{n-2} \alpha_{i} \frac{\eta_{i}^{4}-\zeta_{i}^{4}}{4}\right\}\right]} \leq 1 .
$$

In view of $\left(\mathrm{A}_{4}\right)$, there exists $M$ such that $\|x\| \neq M$. Let us set

$$
U=\{x \in C([0,1], X):\|x\|<M+1\} .
$$

Note that the operator $S: \bar{U} \rightarrow C([0,1], \mathbb{R})$ is continuous and completely continuous. From the choice of $U$, there is no $x \in \partial U$ such that $x=\lambda S(x)$ for some $\lambda \in(0,1)$. Consequently, by the nonlinear alternative of Leray-Schauder type (Lemma 2.2), we deduce that $S$ has a fixed point $x \in \bar{U}$ which is a solution of the problem (1.2). This completes the proof.

Now we make use of Leray-Schauder degree theory to establish our existence result.

Theorem 3.5 Let $f:[0,1] \times \mathbb{R} \rightarrow \mathbb{R}$. Assume that there exist constants $0 \leq \kappa<\frac{1}{\Lambda}$, where $\Lambda$ is given by (3.6) and $K>0$ such that $|f(t, x)| \leq \kappa\|x\|+K$ for all $t \in[0,1], x \in \mathbb{R}$. Then the boundary value problem (1.2) has at least one solution.

Proof In view of the fixed point problem (3.4), we just need to prove the existence of at least one solution $x \in \mathbb{R}$ satisfying (3.4). Define a suitable ball $B_{R} \subset C[0,1]$ with radius $R>0$ as

$$
B_{R}=\{x \in \mathcal{C}:\|x\|<R\}
$$

where $R$ will be fixed later. Then it is sufficient to show that $S: \bar{B}_{R} \rightarrow \mathcal{C}$ satisfies

$$
x \neq \lambda S x, \quad \forall x \in \partial B_{R} \text { and } \forall \lambda \in[0,1] .
$$

Let us set

$$
H(\lambda, x)=\lambda S x, \quad x \in \mathcal{C}, \lambda \in[0,1] .
$$

Then, by the Arzelá-Ascoli theorem, $h_{\lambda}(x)=x-H(\lambda, x)=x-\lambda S x$ is completely continuous. If (3.8) is true, then the following Leray-Schauder degrees are well defined and by the homotopy invariance of topological degree, it follows that

$$
\begin{aligned}
\operatorname{deg}\left(h_{\lambda}, B_{R}, 0\right) & =\operatorname{deg}\left(I-\lambda S, B_{R}, 0\right)=\operatorname{deg}\left(h_{1}, B_{R}, 0\right) \\
& =\operatorname{deg}\left(h_{0}, B_{R}, 0\right)=\operatorname{deg}\left(I, B_{R}, 0\right)=1 \neq 0, \quad 0 \in B_{r},
\end{aligned}
$$

where $I$ denotes the unit operator. By the nonzero property of Leray-Schauder degree, $h_{1}(x)=x-S x=0$ for at least one $x \in B_{R}$. In order to prove (3.8), we assume that $x=\lambda S x$ 
for some $\lambda \in[0,1]$ and for all $t \in[0,1]$ so that

$$
\begin{aligned}
|x(t)|= & |\lambda(S x)(t)| \\
\leq & \int_{0}^{t} \frac{(t-s)^{2}}{2}|f(s, x(s))| d s+|\vartheta| t^{2}\left[\int_{0}^{1} \frac{(1-s)^{2}}{2}|f(s, x(s))| d s\right. \\
& \left.+\sum_{i=1}^{n-2} \alpha_{i} \int_{\zeta_{i}}^{\eta_{i}}\left(\int_{0}^{s} \frac{(s-u)^{2}}{2}|f(u, x(u))| d u\right) d s\right] \\
\leq & (\kappa\|x\|+K)\left\{\frac{1}{2} \int_{0}^{t}(t-s)^{2} d s\right. \\
& \left.+|\vartheta| t^{2}\left[\int_{0}^{1} \frac{(1-s)^{2}}{2} d s+\sum_{i=1}^{n-2} \alpha_{i} \int_{\zeta_{i}}^{\eta_{i}}\left(\int_{0}^{s} \frac{(s-u)^{2}}{2} d u\right) d s\right]\right\} \\
\leq & \frac{(\kappa\|x\|+K)\left[1+|\vartheta|\left\{1+\sum_{i=1}^{n-2} \frac{\alpha_{i}\left(\eta_{i}^{4}-\zeta_{i}^{4}\right)}{4}\right\}\right]}{6} \quad(\kappa\|x\|+K) \Lambda,
\end{aligned}
$$

which, on taking the norm $\left(\sup _{t \in[0,1]}|x(t)|=\|x\|\right)$ and solving for $\|x\|$, yields

$$
\|x\| \leq \frac{K \Lambda}{1-\kappa \Lambda}
$$

Letting $R=\frac{K \Lambda}{1-\kappa \Lambda}+1$, (3.8) holds. This completes the proof.

Now we present an existence result via nonlinear contractions.

Definition 3.6 Let $E$ be a Banach space and let $\mathcal{V}: E \rightarrow E$ be a mapping. $\mathcal{V}$ is said to be a nonlinear contraction if there exists a continuous nondecreasing function $\Psi: \mathbb{R}^{+} \rightarrow \mathbb{R}^{+}$ such that $\Psi(0)=0$ and $\Psi(\xi)<\xi$ for all $\xi>0$ with the property:

$$
\|\mathcal{V} x-\mathcal{V} y\| \leq \Psi(\|x-y\|), \quad \forall x, y \in E .
$$

Lemma 3.7 (Boyd and Wong) Let $E$ be a Banach space and let $\mathcal{V}: E \rightarrow E$ be a nonlinear contraction. Then $\mathcal{V}$ has a unique fixed point in $E$.

Theorem 3.8 Assume that:

$\left(\mathrm{A}_{5}\right)|f(t, x)-f(t, y)| \leq h(t) \frac{|x-y|}{H^{*}+|x-y|}, t \in[0,1], x, y \geq 0$, where $h:[0,1] \rightarrow \mathbb{R}^{+}$is continuous and

$$
\begin{aligned}
H^{*}= & \int_{0}^{1} \frac{(1-s)^{2}}{2} h(s) d s+|\vartheta|\left[\int_{0}^{1} \frac{(1-s)^{2}}{2} h(s) d s\right. \\
& \left.+\sum_{i=1}^{n-2} \alpha_{i} \int_{\zeta_{i}}^{\eta_{i}}\left(\int_{0}^{s} \frac{(s-u)^{2}}{2} h(u) d u\right) d s\right]
\end{aligned}
$$

Then the boundary value problem (1.2) has a unique solution. 
Proof We define the operator $S: \mathcal{C} \rightarrow \mathcal{C}$ by (3.5).

Let a continuous nondecreasing function $\Psi: \mathbb{R}^{+} \rightarrow \mathbb{R}^{+}$satisfying $\Psi(0)=0$ and $\Psi(\xi)<\xi$ for all $\xi>0$ be defined by

$$
\Psi(\xi)=\frac{H^{*} \xi}{H^{*}+\xi}, \quad \forall \xi \geq 0
$$

Let $x, y \in \mathcal{C}$. Then

$$
|f(s, x(s))-f(s, y(s))| \leq \frac{h(s)}{H^{*}} \Psi(\|x-y\|)
$$

so that

$$
\begin{aligned}
|S x(t)-S y(t)| \leq & \int_{0}^{t} \frac{(1-s)^{2}}{2} h(s) \frac{|x(s)-y(s)|}{H^{*}+|x(s)-y(s)|} d s \\
& +|\vartheta| \int_{0}^{1} \frac{(1-s)^{2}}{2} h(s) \frac{|x(s)-y(s)|}{H^{*}+|x(s)-y(s)|} d s \\
& +|\vartheta| \sum_{i=1}^{n-2} \alpha_{i} \int_{\zeta_{i}}^{\eta_{i}}\left(\int_{0}^{s} \frac{(s-u)^{2}}{2} h(m) \frac{|x(m)-y(m)|}{H^{*}+|x(m)-y(m)|} d m\right) d s,
\end{aligned}
$$

for $t \in[0,1]$. In view of (3.9), it follows that $\|S x-S y\| \leq \Psi(\|x-y\|)$ and hence $S$ is a nonlinear contraction. Thus, by Lemma 3.7, the operator $S$ has a unique fixed point in $\mathcal{C}$, which in turn is a unique solution of problem (1.2).

Example 3.9 Consider the following boundary value problem

$$
\left\{\begin{array}{l}
x^{\prime \prime \prime}(t)=f(t, x(t)), \quad t \in[0,1], \\
x(0)=0, \quad x^{\prime}(0)=0, \quad x(1)=\sum_{i=1}^{3} \alpha_{i} \int_{\zeta_{i}}^{\eta_{i}} x(s) d s,
\end{array}\right.
$$

where $\zeta_{1}=1 / 16, \zeta_{2}=5 / 16, \zeta_{3}=9 / 16, \eta_{1}=1 / 4, \eta_{2}=1 / 2, \eta_{3}=3 / 4, \alpha_{1}=1 / 3, \alpha_{2}=2 / 3, \alpha_{3}=1$.

With the given data we find $\theta=1.1160762, \Lambda=0.3644399$.

(a) Let $f:[0,1] \times \mathbb{R} \rightarrow \mathbb{R}$ be defined by

$$
f(t, x)=L\left(1+\sin t+e^{-t} \tan ^{-1} x(t)\right), \quad 0<t<1 .
$$

Clearly

$$
|f(t, x)-f(t, y)| \leq L e^{-t}\left|\tan ^{-1} x-\tan ^{-1} y\right| \leq L|x-y| .
$$

Fixing $L<\frac{1}{\Lambda} \approx 2.7439366$, all the conditions of Theorem 3.2 hold. Hence, the problem (3.10) with $f$ defined by (3.11) has a unique solution.

(b) Let us choose

$$
f(t, x)=\frac{1}{4} \sin \left(\frac{4 \pi}{3} x\right)+\frac{(x+1)^{2}}{3+(x+1)^{2}}, \quad 0<t<1
$$


and note that

$$
|f(t, x)| \leq \frac{\pi}{3}|x|+1
$$

with $\kappa=\frac{\pi}{3}<\frac{1}{\Lambda} \approx 2.7439366$. Thus, the conclusion of Theorem 3.5 applies to the problem (3.10) with $f$ defined by (3.12).

\section{Problem III}

Definition 4.1 A function $x \in C^{(3)}([0,1], \mathbb{R})$ is a solution of the problem (1.3) if there exists a function $f \in L^{1}([0,1], \mathbb{R})$ such that $f(t) \in F(t, x(t))$ a.e. on $[0,1]$ and

$$
\begin{aligned}
x(t)= & \int_{0}^{t} \frac{(t-s)^{2}}{2} f(s) d s-\vartheta t^{2}\left[\int_{0}^{1} \frac{(1-s)^{2}}{2} f(s) d s\right. \\
& \left.-\sum_{i=1}^{n-2} \alpha_{i} \int_{\zeta_{i}}^{\eta_{i}}\left(\int_{0}^{s} \frac{(s-u)^{2}}{2} f(u) d u\right) d s\right] .
\end{aligned}
$$

\subsection{The Carathéodory case}

In this subsection, we are concerned with the existence of solutions for the problem (1.3) when the right hand side has convex values. We first recall some preliminary facts.

For a normed space $(X,\|\cdot\|)$, let $\mathcal{P}_{c l}(X)=\{Y \in \mathcal{P}(X): Y$ is closed $\}, P_{b}(X)=\{Y \in$ $\mathcal{P}(X): Y$ is bounded $\}, \mathcal{P}_{c p}(X)=\{Y \in \mathcal{P}(X): Y$ is compact $\}$, and $\mathcal{P}_{c p, c}(X)=\{Y \in \mathcal{P}(X)$ : $Y$ is compact and convex .

Definition 4.2 A multi-valued map $G: X \rightarrow \mathcal{P}(X)$ :

(i) is convex (closed) valued if $G(x)$ is convex (closed) for all $x \in X$;

(ii) is bounded on bounded sets if $G(\mathbb{B})=\bigcup_{x \in \mathbb{B}} G(x)$ is bounded in $X$ for all $\mathbb{B} \in \mathcal{P}_{b}(X)$ (i.e. $\sup _{x \in \mathbb{B}}\{\sup \{|y|: y \in G(x)\}\}<\infty$ );

(iii) is called upper semicontinuous (u.s.c.) on $X$ if for each $x_{0} \in X$, the set $G\left(x_{0}\right)$ is a nonempty closed subset of $X$, and if for each open set $N$ of $X$ containing $G\left(x_{0}\right)$, there exists an open neighborhood $\mathcal{N}_{0}$ of $x_{0}$ such that $G\left(\mathcal{N}_{0}\right) \subseteq N$;

(v) is said to be completely continuous if $G(\mathbb{B})$ is relatively compact for every $\mathbb{B} \in P_{b}(X)$;

(v) has a fixed point if there is $x \in X$ such that $x \in G(x)$. The fixed point set of the multi-valued operator $G$ will be denoted by Fix $G$.

Remark 4.3 It is well known that, if the multi-valued map $G$ is completely continuous with nonempty compact values, then $G$ is u.s.c. if and only if $G$ has a closed graph, i.e., $x_{n} \rightarrow x_{*}, y_{n} \rightarrow y_{*}, y_{n} \in G\left(x_{n}\right)$ imply $y_{*} \in G\left(x_{*}\right)$.

Definition 4.4 A multi-valued map $G:[0 ; 1] \rightarrow P_{c l}(\mathbb{R})$ is said to be measurable if for every $y \in \mathbb{R}$, the function

$$
t \longmapsto d(y, G(t))=\inf \{\|y-z\|: z \in G(t)\}
$$

is measurable.

Definition 4.5 A multi-valued map $F:[0,1] \times \mathbb{R} \rightarrow \mathcal{P}(\mathbb{R})$ is said to be Carathéodory if (i) $t \longmapsto F(t, x)$ is measurable for each $x \in \mathbb{R}$; 
(ii) $x \longmapsto F(t, x)$ is upper semicontinuous for almost all $t \in[0,1]$; Further a Carathéodory function $F$ is called $L^{1}$-Carathéodory if

(iii) for each $\alpha>0$, there exists $\varphi_{\alpha} \in L^{1}\left([0,1], \mathbb{R}^{+}\right)$such that

$$
\|F(t, x)\|=\sup \{|v|: v \in F(t, x)\} \leq \varphi_{\alpha}(t)
$$

for all $\|x\| \leq \alpha$ and for a.e. $t \in[0,1]$.

For each $y \in C([0,1], \mathbb{R})$, define the set of selections of $F$ by

$$
S_{F, y}:=\left\{v \in L^{1}([0,1], \mathbb{R}): v(t) \in F(t, y(t)) \text { for a.e. } t \in[0,1]\right\} .
$$

The consideration of this subsection is based on the following lemmas.

Lemma 4.6 (Nonlinear alternative for Kakutani maps) Let E be a Banach space, $C$ a closed convex subset of $E, U$ an open subset of $C$ and $0 \in U$. Suppose that $F: \bar{U} \rightarrow \mathcal{P}_{c p, c}(C)$ is a upper semicontinuous compact map; here $\mathcal{P}_{c p, c}(C)$ denotes the family of nonempty, compact convex subsets of $C$. Then either

(i) F has a fixed point in $\bar{U}$, or

(ii) there is $a u \in \partial U$ and $\lambda \in(0,1)$ with $u \in \lambda F(u)$.

Lemma 4.7 Let $X$ be a Banach space. Let $F:[0,1] \times \mathbb{R} \rightarrow \mathcal{P}_{c p, c}(\mathbb{R})$ be an $L^{1}$-Carathéodory multi-valued map and let $\Theta$ be a linear continuous mapping from $L^{1}([0,1], \mathbb{R})$ to $C([0$, $1], \mathbb{R})$. Then the operator

$$
\Theta \circ S_{F}: C([0,1], \mathbb{R}) \rightarrow P_{c p, c}(C([0,1], \mathbb{R})), \quad x \mapsto\left(\Theta \circ S_{F}\right)(x)=\Theta\left(S_{F, x}\right)
$$

is a closed graph operator in $C([0,1], \mathbb{R}) \times C([0,1], \mathbb{R})$.

\section{Theorem 4.8 Assume that}

$\left(\mathrm{H}_{1}\right) \quad F:[0,1] \times \mathbb{R} \rightarrow \mathcal{P}(\mathbb{R})$ is Carathéodory and has nonempty compact convex values;

$\left(\mathrm{H}_{2}\right)$ there exists a continuous nondecreasing function $\psi:[0, \infty) \rightarrow(0, \infty)$ and a function $p \in C\left([0,1], \mathbb{R}^{+}\right)$such that

$$
\|F(t, x)\|_{\mathcal{P}}:=\sup \{|y|: y \in F(t, x)\} \leq p(t) \psi(\|x\|) \quad \text { for each }(t, x) \in[0,1] \times \mathbb{R} ;
$$

$\left(\mathrm{H}_{3}\right)$ there exists a number $M>0$ such that

$$
\frac{M}{\frac{\|p\| \psi(M)}{6}\left[1+|\vartheta|\left\{1+\sum_{i=1}^{n-2} \alpha_{i} \frac{\eta_{i}^{4}-\zeta_{i}^{4}}{4}\right\}\right]}>1 .
$$

Then the boundary value problem (1.3) has at least one solution on $[0,1]$.

Proof Define an operator $\Omega: C([0,1], \mathbb{R}) \rightarrow \mathcal{P}(C([0,1], \mathbb{R}))$ by

$$
\Omega(x)=\left\{\begin{array}{l}
h \in C([0,1], \mathbb{R}): \\
h(t)= \begin{cases}\int_{0}^{t} \frac{(t-s)^{2}}{2} f(s) d s \\
-\vartheta t^{2}\left[\int_{0}^{1} \frac{(1-s)^{2}}{2} f(s) d s\right. \\
\left.-\sum_{i=1}^{n-2} \alpha_{i} \int_{\zeta_{i}}^{\eta_{i}}\left(\int_{0}^{s} \frac{(s-u)^{2}}{2} f(u) d u\right) d s\right], \quad 0 \leq t \leq 1,\end{cases}
\end{array}\right.
$$


for $f \in S_{F, x}$. We will show that $\Omega$ satisfies the assumptions of the nonlinear alternative of Leray-Schauder type. The proof consists of several steps. As a first step, we show that $\Omega$ is convex for each $x \in C([0,1], \mathbb{R})$. For that, let $h_{1}, h_{2} \in \Omega(x)$. Then there exist $f_{1}, f_{2} \in S_{F, x}$ such that, for each $t \in[0,1]$, we have

$$
\begin{aligned}
h_{i}(t)= & \int_{0}^{t} \frac{(t-s)^{2}}{2} f_{i}(s) d s-\vartheta t^{2}\left[\int_{0}^{1} \frac{(1-s)^{2}}{2} f_{i}(s) d s\right. \\
& \left.-\sum_{i=1}^{n-2} \alpha_{i} \int_{\zeta_{i}}^{\eta_{i}}\left(\int_{0}^{s} \frac{(s-u)^{2}}{2} f_{i}(u) d u\right) d s\right], \quad i=1,2 .
\end{aligned}
$$

Let $0 \leq \omega \leq 1$. Then, for each $t \in[0,1]$, we have

$$
\begin{aligned}
{\left[\omega h_{1}+(1-\omega) h_{2}\right](t)=} & \int_{0}^{t} \frac{(t-s)^{2}}{2}\left[\omega f_{1}(s)+(1-\omega) f_{2}(s)\right] d s \\
& -\vartheta t^{2}\left[\int_{0}^{1} \frac{(1-s)^{2}}{2}\left[\omega f_{1}(s)+(1-\omega) f_{2}(s)\right] d s\right. \\
& \left.-\sum_{i=1}^{n-2} \alpha_{i} \int_{\zeta_{i}}^{\eta_{i}}\left(\int_{0}^{s} \frac{(s-u)^{2}}{2}\left[\omega f_{1}(s)+(1-\omega) f_{2}(s)\right] d u\right) d s\right]
\end{aligned}
$$

Since $S_{F, x}$ is convex ( $F$ has convex values), $\omega h_{1}+(1-\omega) h_{2} \in \Omega(x)$.

Next, we show that $\Omega$ maps bounded sets into bounded sets in $C([0,1], \mathbb{R})$. For a positive number $r$, let $B_{r}=\{x \in C([0,1], \mathbb{R}):\|x\| \leq r\}$ be a bounded set in $C([0,1], \mathbb{R})$. Then, for each $h \in \Omega(x), x \in B_{r}$, there exists $f \in S_{F, x}$ such that

$$
\begin{aligned}
h(t)= & \int_{0}^{t} \frac{(t-s)^{2}}{2} f(s) d s-\vartheta t^{2}\left[\int_{0}^{1} \frac{(1-s)^{2}}{2} f(s) d s\right. \\
& \left.-\sum_{i=1}^{n-2} \alpha_{i} \int_{\zeta_{i}}^{\eta_{i}}\left(\int_{0}^{s} \frac{(s-u)^{2}}{2} f(u) d u\right) d s\right] .
\end{aligned}
$$

Then, as in Theorem 3.4,

$$
\begin{aligned}
|h(t)| \leq & \int_{0}^{t} \frac{(t-s)^{2}}{2}|f(s)| d s+|\vartheta| t^{2} \int_{0}^{1} \frac{(1-s)^{2}}{2}|f(s)| d s \\
& +|\vartheta| t^{2} \sum_{i=1}^{n-2} \alpha_{i} \int_{\zeta_{i}}^{\eta_{i}}\left(\int_{0}^{s} \frac{(s-u)^{2}}{2}|f(u)| d u\right) d s \\
\leq & \frac{\|p\| \psi(\|x\|)}{6}\left[1+|\vartheta|\left\{1+\sum_{i=1}^{n-2} \alpha_{i} \frac{\eta_{i}^{4}-\zeta_{i}^{4}}{4}\right\}\right] .
\end{aligned}
$$

Thus,

$$
\|h\| \leq \frac{\|p\| \psi(\|x\|)}{6}\left[1+|\vartheta|\left\{1+\sum_{i=1}^{n-2} \alpha_{i} \frac{\eta_{i}^{4}-\zeta_{i}^{4}}{4}\right\}\right] .
$$

Now we show that $\Omega$ maps bounded sets into equicontinuous sets of $C([0,1], \mathbb{R})$. Let $t^{\prime}, t^{\prime \prime} \in$ $[0,1]$ with $t^{\prime}<t^{\prime \prime}$ and $x \in B_{r}$, where $B_{r}$ is a bounded set of $C([0,1], \mathbb{R})$. For each $h \in \Omega(x)$, 
we obtain

$$
\begin{aligned}
\left|h\left(t^{\prime \prime}\right)-h\left(t^{\prime}\right)\right|= & \mid \frac{1}{2} \int_{0}^{t^{\prime \prime}}\left(t^{\prime \prime}-s\right)^{2} f(s) d s-\frac{1}{2} \int_{0}^{t^{\prime}}\left(t^{\prime}-s\right)^{2} f(s) d s \\
& -\vartheta\left(\left(t^{\prime \prime}\right)^{2}-\left(t^{\prime}\right)^{2}\right)\left[\int_{0}^{1} \frac{(1-s)^{2}}{2} f(s) d s\right. \\
& \left.-\sum_{i=1}^{n-2} \alpha_{i} \int_{\zeta_{i}}^{\eta_{i}}\left(\int_{0}^{s} \frac{(s-u)^{2}}{2} f(u) d u\right) d s\right] \mid \\
\leq & \left|\frac{1}{2} \int_{0}^{t^{\prime}}\left[\left(t^{\prime \prime}-s\right)^{2}-\left(t^{\prime}-s\right)^{2}\right] \psi(r) p(s) d s\right| \\
& +\left|\frac{1}{2} \int_{t^{\prime}}^{t^{\prime \prime}}\left(t^{\prime \prime}-s\right)^{2} \psi(r) p(s) d s\right| \\
& +\mid \vartheta\left(\left(t^{\prime \prime}\right)^{2}-\left(t^{\prime}\right)^{2}\right)\left[\int_{0}^{1} \frac{(1-s)^{q-1}}{2} \psi(r) p(s) d s\right. \\
& \left.+\sum_{i=1}^{n-2} \alpha_{i} \int_{\zeta_{i}}^{\eta_{i}}\left(\int_{0}^{s} \frac{(s-u)^{2}}{2} \psi(r) p(u) d u\right) d s\right] .
\end{aligned}
$$

Obviously the right hand side of the above inequality tends to zero independently of $x \in B_{r}$ as $t^{\prime \prime}-t^{\prime} \rightarrow 0$. As $\Omega$ satisfies the above three assumptions, by the Arzelá-Ascoli theorem $\Omega: C([0,1], \mathbb{R}) \rightarrow \mathcal{P}(C([0,1], \mathbb{R}))$ is completely continuous.

In our next step, we show that $\Omega$ has a closed graph. Let $x_{n} \rightarrow x_{*}, h_{n} \in \Omega\left(x_{n}\right)$ and $h_{n} \rightarrow$ $h_{*}$. Then we need to show that $h_{*} \in \Omega\left(x_{*}\right)$. Associated with $h_{n} \in \Omega\left(x_{n}\right)$, there exists $f_{n} \in$ $S_{F, x_{n}}$ such that, for each $t \in[0,1]$,

$$
\begin{aligned}
h_{n}(t)= & \int_{0}^{t} \frac{(t-s)^{2}}{2} f_{n}(s) d s-\vartheta t^{2}\left[\int_{0}^{1} \frac{(1-s)^{2}}{2} f_{n}(s) d s\right. \\
& \left.-\sum_{i=1}^{n-2} \alpha_{i} \int_{\zeta_{i}}^{\eta_{i}}\left(\int_{0}^{s} \frac{(s-u)^{2}}{2} f_{n}(u) d u\right) d s\right] .
\end{aligned}
$$

Thus we have to show that there exists $f_{*} \in S_{F, x_{*}}$ such that, for each $t \in[0,1]$,

$$
\begin{aligned}
h_{*}(t)= & \int_{0}^{t} \frac{(t-s)^{2}}{2} f_{*}(s) d s-\vartheta t^{2}\left[\int_{0}^{1} \frac{(1-s)^{2}}{2} f_{*}(s) d s\right. \\
& \left.-\sum_{i=1}^{n-2} \alpha_{i} \int_{\zeta_{i}}^{\eta_{i}}\left(\int_{0}^{s} \frac{(s-u)^{2}}{2} f_{*}(u) d u\right) d s\right] .
\end{aligned}
$$

Let us consider the continuous linear operator $\Theta: L^{1}([0,1], \mathbb{R}) \rightarrow C([0,1], \mathbb{R})$ given by

$$
\begin{aligned}
f \mapsto \Theta(f)= & \int_{0}^{t} \frac{(t-s)^{2}}{2} f(s) d s-\vartheta t^{2}\left[\int_{0}^{1} \frac{(1-s)^{2}}{2} f(s) d s\right. \\
& \left.-\sum_{i=1}^{n-2} \alpha_{i} \int_{\zeta_{i}}^{\eta_{i}}\left(\int_{0}^{s} \frac{(s-u)^{2}}{2} f(u) d u\right) d s\right] .
\end{aligned}
$$


Observe that

$$
\begin{aligned}
\left\|h_{n}(t)-h_{*}(t)\right\|= & \| \int_{0}^{t} \frac{(t-s)^{2}}{2}\left(f_{n}(s)-f_{*}(s)\right) d s \\
& -\vartheta t^{2}\left[\int_{0}^{1} \frac{(1-s)^{2}}{2}\left(f_{n}(s)-f_{*}(s)\right) d s\right. \\
& \left.-\sum_{i=1}^{n-2} \alpha_{i} \int_{\zeta_{i}}^{\eta_{i}}\left(\int_{0}^{s} \frac{(s-u)^{2}}{2}\left(f_{n}(u)-f_{*}(u)\right) d u\right) d s\right] \| \rightarrow 0,
\end{aligned}
$$

as $n \rightarrow \infty$. Thus, it follows by Lemma 4.7 that $\Theta \circ S_{F}$ is a closed graph operator. Further, we have $h_{n}(t) \in \Theta\left(S_{F, x_{n}}\right)$. Since $x_{n} \rightarrow x_{*}$, we have

$$
\begin{aligned}
h_{*}(t)= & \int_{0}^{t} \frac{(t-s)^{2}}{2} f_{*}(s) d s-\vartheta t^{2}\left[\int_{0}^{1} \frac{(1-s)^{2}}{2} f_{*}(s) d s\right. \\
& \left.-\sum_{i=1}^{n-2} \alpha_{i} \int_{\zeta_{i}}^{\eta_{i}}\left(\int_{0}^{s} \frac{(s-u)^{2}}{2} f_{*}(u) d u\right) d s\right],
\end{aligned}
$$

for some $f_{*} \in S_{F, x_{*}}$.

Finally, we discuss a priori bounds on the solutions. Let $x$ be a solution of (1.3). Then there exists $f \in L^{1}([0,1], \mathbb{R})$ with $f \in S_{F, x}$ such that, for $t \in[0,1]$, we have

$$
\begin{aligned}
x(t)= & \int_{0}^{t} \frac{(t-s)^{2}}{2} f(s) d s-\vartheta t^{2}\left[\int_{0}^{1} \frac{(1-s)^{2}}{2} f(s) d s\right. \\
& \left.-\sum_{i=1}^{n-2} \alpha_{i} \int_{\zeta_{i}}^{\eta_{i}}\left(\int_{0}^{s} \frac{(s-u)^{2}}{2} f(u) d u\right) d s\right] .
\end{aligned}
$$

In view of $\left(\mathrm{H}_{2}\right)$, and using the computations in the second step above, for each $t \in[0,1]$, we obtain

$$
\begin{aligned}
|x(t)| \leq & \int_{0}^{t} \frac{(t-s)^{2}}{2}|f(s)| d s+|\vartheta| t^{2} \int_{0}^{1} \frac{(1-s)^{2}}{2}|f(s)| d s \\
& +|\vartheta| t^{2} \sum_{i=1}^{n-2} \alpha_{i} \int_{\zeta_{i}}^{\eta_{i}}\left(\int_{0}^{s} \frac{(s-u)^{2}}{2}|f(u)| d u\right) d s \\
\leq & \frac{\|p\| \psi(\|x\|)}{6}\left[1+|\vartheta|\left\{1+\sum_{i=1}^{n-2} \alpha_{i} \frac{\eta_{i}^{4}-\zeta_{i}^{4}}{4}\right\}\right] .
\end{aligned}
$$

Consequently, we have

$$
\frac{\|x\|}{\frac{\|p\| \psi(\|x\|)}{6}\left[1+|\vartheta|\left\{1+\sum_{i=1}^{n-2} \alpha_{i} \frac{\eta_{i}^{4}-\zeta_{i}^{4}}{4}\right\}\right]} \leq 1 .
$$

In view of $\left(\mathrm{H}_{3}\right)$, there exists $M$ such that $\|x\| \neq M$. Let us set

$$
U=\{x \in C([0,1], \mathbb{R}):\|x\|<M+1\} .
$$


Note that the operator $\Omega: \bar{U} \rightarrow \mathcal{P}(C([0,1], \mathbb{R}))$ is upper semicontinuous and completely continuous. From the choice of $U$, there is no $x \in \partial U$ such that $x \in \mu \Omega(x)$ for some $\mu \in$ $(0,1)$. Consequently, by the nonlinear alternative of Leray-Schauder type (Lemma 4.6), we deduce that $\Omega$ has a fixed point $x \in \bar{U}$ which is a solution of the problem (1.3). This completes the proof.

\subsection{The lower semicontinuous case}

Here, we study the case when $F$ is not necessarily convex valued. Our strategy to deal with this problems is based on the nonlinear alternative of Leray-Schauder type together with the selection theorem of Bressan and Colombo for lower semicontinuous maps with decomposable values.

Definition 4.9 Let $X$ be a nonempty closed subset of a Banach space $E$ and $G: X \rightarrow \mathcal{P}(E)$ be a multi-valued operator with nonempty closed values. $G$ is lower semicontinuous (l.s.c.) if the set $\{y \in X: G(y) \cap B \neq \emptyset\}$ is open for any open set $B$ in $E$.

Definition 4.10 Let $A$ be a subset of [0,1] $\times \mathbb{R} . A$ is $\mathcal{L} \otimes \mathcal{B}$ measurable if $A$ belongs to the $\sigma$-algebra generated by all sets of the form $\mathcal{J} \times \mathcal{D}$, where $\mathcal{J}$ is Lebesgue measurable in $[0,1]$ and $\mathcal{D}$ is Borel measurable in $\mathbb{R}$.

Definition 4.11 A subset $\mathcal{A}$ of $L^{1}([0,1], \mathbb{R})$ is decomposable if for all $x, y \in \mathcal{A}$ and measurable $\mathcal{J} \subset[0,1]=J$, the function $x_{\chi_{\mathcal{J}}}+y \chi_{\mathcal{J}-\mathcal{J}} \in \mathcal{A}$, where $\chi_{\mathcal{J}}$ stands for the characteristic function of $\mathcal{J}$.

Definition 4.12 Let $Y$ be a separable metric space and let $N: Y \rightarrow \mathcal{P}\left(L^{1}([0,1], \mathbb{R})\right)$ be a multi-valued operator. We say $N$ has a property (BC) if $N$ is lower semicontinuous (l.s.c.) and has nonempty closed and decomposable values.

Let $F:[0,1] \times \mathbb{R} \rightarrow \mathcal{P}(\mathbb{R})$ be a multi-valued map with nonempty compact values. Define a multi-valued operator $\mathcal{F}: C([0,1] \times \mathbb{R}) \rightarrow \mathcal{P}\left(L^{1}([0,1], \mathbb{R})\right)$ associated with $F$ as

$$
\mathcal{F}(x)=\left\{w \in L^{1}([0,1], \mathbb{R}): w(t) \in F(t, x(t)) \text { for a.e. } t \in[0,1]\right\}
$$

which is called the Nemytskii operator associated with $F$.

Definition 4.13 Let $F:[0,1] \times \mathbb{R} \rightarrow \mathcal{P}(\mathbb{R})$ be a multi-valued function with nonempty compact values. We say $F$ is of lower semicontinuous type (l.s.c. type) if its associated Nemytskii operator $\mathcal{F}$ is lower semicontinuous and has nonempty closed and decomposable values.

Lemma 4.14 (Bressan and Colombo) Let $Y$ be a separable metric space and let $N: Y \rightarrow$ $\mathcal{P}\left(L^{1}([0,1], \mathbb{R})\right)$ be a multi-valued operator satisfying the property $(B C)$. Then $N$ has a continuous selection, that is, there exists a continuous function (single-valued) $g: Y \rightarrow$ $L^{1}([0,1], \mathbb{R})$ such that $g(x) \in N(x)$ for every $x \in Y$.

Theorem 4.15 Assume that $\left(\mathrm{H}_{2}\right),\left(\mathrm{H}_{3}\right)$ and the following condition holds:

$\left(\mathrm{H}_{4}\right) \quad F:[0,1] \times \mathbb{R} \rightarrow \mathcal{P}(\mathbb{R})$ is a nonempty compact-valued multi-valued map such that 
(a) $(t, x) \longmapsto F(t, x)$ is $\mathcal{L} \otimes \mathcal{B}$ measurable,

(b) $x \longmapsto F(t, x)$ is lower semicontinuous for each $t \in[0,1]$.

Then the boundary value problem (1.3) has at least one solution on $[0,1]$.

Proof It follows from $\left(\mathrm{H}_{2}\right)$ and $\left(\mathrm{H}_{4}\right)$ that $F$ is of 1.s.c. type. Then, by Lemma 4.14, there exists a continuous function $f: C^{(3)}([0,1], \mathbb{R}) \rightarrow L^{1}([0,1], \mathbb{R})$ such that $f(x) \in \mathcal{F}(x)$ for all $x \in C([0,1], \mathbb{R})$.

Consider the problem

$$
\left\{\begin{array}{l}
x^{\prime \prime \prime}(t)=f(x(t)), \quad 0<t<1, \\
x(0)=0, \quad x^{\prime}(0)=0, \\
x(1)=\sum_{i=1}^{n-2} \alpha_{i} \int_{\zeta_{i}}^{\eta_{i}} x(s) d s, \quad 0<\zeta_{i}<\eta_{i}<1, i=1,2, \ldots, n-2,
\end{array}\right.
$$

in the space $C([0,1], \mathbb{R})$. It is clear that if $x$ is a solution of the problem (4.2), then $x$ is a solution to the problem (1.3). In order to transform the problem (4.2) into a fixed point problem, we define the operator $\bar{\Omega}$ as

$$
\bar{\Omega} x(t)=\left\{\begin{array}{c}
\frac{1}{2} \int_{0}^{t}(t-s)^{2} f(x(s)) d s-\vartheta t^{2}\left[\int_{0}^{1} \frac{(1-s)^{2}}{2} f(x(s)) d s\right. \\
\left.-\sum_{i=1}^{n-2} \alpha_{i} \int_{\zeta_{i}}^{\eta_{i}}\left(\int_{0}^{s} \frac{(s-u)^{2}}{2} f(x(u)) d u\right) d s\right], \quad 0 \leq t \leq 1 .
\end{array}\right.
$$

It can easily be shown that $\bar{\Omega}$ is continuous and completely continuous. The remaining part of the proof is similar to that of Theorem 4.8. So we omit it. This completes the proof.

\subsection{The Lipschitz case}

Now we prove the existence of solutions for the problem (1.3) with a non-convex valued right hand side by applying a fixed point theorem for multi-valued maps due to Covitz and Nadler.

Let $(X, d)$ be a metric space induced from the normed space $(X ;\|\cdot\|)$. Consider $H_{d}$ : $\mathcal{P}(X) \times \mathcal{P}(X) \rightarrow \mathbb{R} \cup\{\infty\}$ given by

$$
H_{d}(A, B)=\max \left\{\sup _{a \in A} d(a, B), \sup _{b \in B} d(A, b)\right\}
$$

where $d(A, b)=\inf _{a \in A} d(a ; b)$ and $d(a, B)=\inf _{b \in B} d(a ; b)$. Then $\left(\mathcal{P}_{b, c l}(X), H_{d}\right)$ is a metric space and $\left(\mathcal{P}_{c l}(X), H_{d}\right)$ is a generalized metric space.

Definition 4.16 A multi-valued operator $N: X \rightarrow \mathcal{P}_{c l}(X)$ is called:

(a) $\gamma$-Lipschitz if and only if there exists $\gamma>0$ such that

$$
H_{d}(N(x), N(y)) \leq \gamma d(x, y) \quad \text { for each } x, y \in X
$$

(b) a contraction if and only if it is $\gamma$-Lipschitz with $\gamma<1$.

Lemma 4.17 (Covitz-Nadler) Let $(X, d)$ be a complete metric space. If $N: X \rightarrow P_{c l}(X)$ is a contraction, then Fix $N \neq \emptyset$. 
Definition 4.18 A measurable multi-valued function $F:[0,1] \rightarrow \mathcal{P}(X)$ is said to be integrably bounded if there exists a function $h \in L^{1}([0,1], X)$ such that, for all $v \in F(t)$, $\|v\| \leq h(t)$ for a.e. $t \in[0,1]$.

Theorem 4.19 Assume that the following conditions hold:

$\left(\mathrm{H}_{5}\right) \quad F:[0,1] \times \mathbb{R} \rightarrow \mathcal{P}_{c p}(\mathbb{R})$ is such that $F(\cdot, x):[0,1] \rightarrow \mathcal{P}_{c p}(\mathbb{R})$ is measurable for each $x \in \mathbb{R}$

$\left(\mathrm{H}_{6}\right) H_{d}(F(t, x), F(t, \bar{x})) \leq m(t)|x-\bar{x}|$ for almost all $t \in[0,1]$ and $x, \bar{x} \in \mathbb{R}$ with $m \in$ $C\left([0,1], \mathbb{R}^{+}\right)$and $d(0, F(t, 0)) \leq m(t)$ for almost all $t \in[0,1]$.

Then the boundary value problem (1.3) has at least one solution on $[0,1]$ if

$$
\frac{\|m\|}{6}\left[1+|\vartheta|\left\{1+\sum_{i=1}^{n-2} \alpha_{i} \frac{\eta_{i}^{4}-\zeta_{i}^{4}}{4}\right\}\right]<1 .
$$

Proof We transform the problem (1.3) into a fixed point problem. Consider the set-valued map $\Omega: C([0,1], \mathbb{R}) \rightarrow \mathcal{P}(C([0,1], \mathbb{R}))$ defined at the beginning of the proof of Theorem 4.8. It is clear that the fixed point of $\Omega$ are solutions of the problem (1.3).

Note that, by the assumption $\left(\mathrm{H}_{5}\right)$, since the set-valued map $F(\cdot, x)$ is measurable, it admits a measurable selection $f:[0,1] \rightarrow \mathbb{R}$. Moreover, from assumption $\left(\mathrm{H}_{6}\right)$

$$
|f(t)| \leq m(t)+m(t)|x(t)|
$$

i.e. $f(\cdot) \in L^{1}([0,1], \mathbb{R})$. Therefore the set $S_{F, x}$ is nonempty. Also note that since $S_{F, x} \neq \emptyset$, $\Omega(x) \neq \emptyset$ for any $x \in C([0,1], \mathbb{R})$.

Now we show that the operator $\Omega$ satisfies the assumptions of Lemma 4.17. To show that $\Omega(x) \in \mathcal{P}_{c l}((C[0,1], \mathbb{R}))$ for each $x \in C([0,1], \mathbb{R})$, let $\left\{u_{n}\right\}_{n \geq 0} \in \Omega(x)$ be such that $u_{n} \rightarrow u$ $(n \rightarrow \infty)$ in $C([0,1], \mathbb{R})$. Then $u \in C([0,1], \mathbb{R})$ and there exists $v_{n} \in S_{F, x}$ such that, for each $t \in[0,1]$, we have

$$
\begin{aligned}
u_{n}(t)= & \int_{0}^{t} \frac{(t-s)^{2}}{2} v_{n}(s) d s-\vartheta t^{2}\left[\int_{0}^{1} \frac{(1-s)^{2}}{2} v_{n}(s) d s\right. \\
& \left.-\sum_{i=1}^{n-2} \alpha_{i} \int_{\zeta_{i}}^{\eta_{i}}\left(\int_{0}^{s} \frac{(s-u)^{2}}{2} v_{n}(u) d u\right) d s\right] .
\end{aligned}
$$

As $F$ has compact values, we may pass onto a subsequence (if necessary) to obtain the result that $v_{n}$ converges to $v$ in $L^{1}([0,1], \mathbb{R})$. Thus, $v \in S_{F, x}$ and, for each $t \in[0,1]$,

$$
\begin{aligned}
u_{n}(t) \rightarrow u(t)= & \int_{0}^{t} \frac{(t-s)^{2}}{2} v(s) d s-\vartheta t^{2}\left[\int_{0}^{1} \frac{(1-s)^{2}}{2} v(s) d s\right. \\
& \left.-\sum_{i=1}^{n-2} \alpha_{i} \int_{\zeta_{i}}^{\eta_{i}}\left(\int_{0}^{s} \frac{(s-u)^{2}}{2} v(u) d u\right) d s\right] .
\end{aligned}
$$

Hence, $u \in \Omega(x)$ and $\Omega(x)$ is closed. 
Next we show that $\Omega$ is a contraction on $C([0,1], \mathbb{R})$, i.e. there exists $\gamma<1$ such that

$$
H_{d}(\Omega(x), \Omega(\bar{x})) \leq \gamma\|x-\bar{x}\| \quad \text { for each } x, \bar{x} \in C^{(3)}([0,1], \mathbb{R})
$$

Let $x, \bar{x} \in C^{(3)}([0,1], \mathbb{R})$ and $h_{1} \in \Omega(x)$. Then there exists $v_{1}(t) \in F(t, x(t))$ such that, for each $t \in[0,1]$,

$$
\begin{aligned}
h_{1}(t)= & \int_{0}^{t} \frac{(t-s)^{2}}{2} v_{1}(s) d s-\vartheta t^{2}\left[\int_{0}^{1} \frac{(1-s)^{2}}{2} v_{1}(s) d s\right. \\
& \left.-\sum_{i=1}^{n-2} \alpha_{i} \int_{\zeta_{i}}^{\eta_{i}}\left(\int_{0}^{s} \frac{(s-u)^{2}}{2} v_{1}(u) d u\right) d s\right] .
\end{aligned}
$$

By $\left(\mathrm{H}_{6}\right)$, we have

$$
H_{d}(F(t, x), F(t, \bar{x})) \leq m(t)|x(t)-\bar{x}(t)| .
$$

So, there exists $w \in F(t, \bar{x}(t))$ such that

$$
\left|v_{1}(t)-w(t)\right| \leq m(t)|x(t)-\bar{x}(t)|, \quad t \in[0,1] .
$$

Define $U:[0,1] \rightarrow \mathcal{P}(\mathbb{R})$ by

$$
U(t)=\left\{w \in \mathbb{R}:\left|v_{1}(t)-w\right| \leq m(t)|x(t)-\bar{x}(t)|\right\} .
$$

Since the multi-valued operator $U(t) \cap F(t, \bar{x}(t))$ is measurable, there exists a function $v_{2}(t)$ which is a measurable selection for $U$. So $v_{2}(t) \in F(t, \bar{x}(t))$ and, for each $t \in[0,1]$, we have $\left|v_{1}(t)-v_{2}(t)\right| \leq m(t)|x(t)-\bar{x}(t)|$.

For each $t \in[0,1]$, let us define

$$
\begin{aligned}
h_{2}(t)= & \int_{0}^{t} \frac{(t-s)^{2}}{2} v_{2}(s) d s-\vartheta t^{2}\left[\int_{0}^{1} \frac{(1-s)^{2}}{2} v_{2}(s) d s\right. \\
& \left.-\sum_{i=1}^{n-2} \alpha_{i} \int_{\zeta_{i}}^{\eta_{i}}\left(\int_{0}^{s} \frac{(s-u)^{2}}{2} v_{2}(u) d u\right) d s\right] .
\end{aligned}
$$

Thus,

$$
\begin{aligned}
\left|h_{1}(t)-h_{2}(t)\right| \leq & \int_{0}^{t} \frac{(t-s)^{2}}{2}\left|v_{1}(s)-v_{2}(s)\right| d s \\
& +|\vartheta| t^{2} \int_{0}^{1} \frac{(1-s)^{2}}{2}\left|v_{1}(s)-v_{2}(s)\right| d s \\
& +|\vartheta| t^{2} \sum_{i=1}^{n-2} \alpha_{i} \int_{\zeta_{i}}^{\eta_{i}}\left(\int_{0}^{s} \frac{(s-u)^{2}}{2}\left|v_{1}(u)-v_{2}(u)\right| d u\right) d s \\
\leq & \frac{\|m\|}{6}\left[1+|\vartheta|\left\{1+\sum_{i=1}^{n-2} \alpha_{i} \frac{\eta_{i}^{4}-\zeta_{i}^{4}}{4}\right\}\right]\|x-\bar{x}\| .
\end{aligned}
$$


Hence,

$$
\left\|h_{1}-h_{2}\right\| \leq \frac{\|m\|}{6}\left[1+|\vartheta|\left\{1+\sum_{i=1}^{n-2} \alpha_{i} \frac{\eta_{i}^{4}-\zeta_{i}^{4}}{4}\right\}\right]\|x-\bar{x}\|
$$

Analogously, interchanging the roles of $x$ and $\bar{x}$, we obtain

$$
\begin{aligned}
H_{d}(\Omega(x), \Omega(\bar{x})) & \leq \gamma\|x-\bar{x}\| \\
& \leq \frac{\|m\|}{6}\left[1+|\vartheta|\left\{1+\sum_{i=1}^{n-2} \alpha_{i} \frac{\eta_{i}^{4}-\zeta_{i}^{4}}{4}\right\}\right]\|x-\bar{x}\| .
\end{aligned}
$$

Since $\Omega$ is a contraction, it follows by Lemma 4.17 that $\Omega$ has a fixed point $x$ which is a solution of (1.3). This completes the proof.

Example 4.20 Consider the following boundary value problem:

$$
\left\{\begin{array}{l}
x^{\prime \prime \prime}(t) \in F(t, x(t)), \quad t \in[0,1], \\
x(0)=0, \quad x^{\prime}(0)=0, \quad x(1)=\sum_{i=1}^{3} \alpha_{i} \int_{\zeta_{i}}^{\eta_{i}} x(s) d s
\end{array}\right.
$$

where $\zeta_{1}=1 / 16, \zeta_{2}=5 / 16, \zeta_{3}=9 / 16, \eta_{1}=1 / 4, \eta_{2}=1 / 2, \eta_{3}=3 / 4, \alpha_{1}=1 / 3, \alpha_{2}=2 / 3, \alpha_{3}=1$.

Let $F:[0,1] \times \mathbb{R} \rightarrow \mathcal{P}(\mathbb{R})$ be a multi-valued map given by

$$
x \rightarrow F(t, x)=\left[\frac{|x|^{5}}{2\left(|x|^{5}+1\right)}+5 t^{3}+4, \frac{|x|}{|x|+1}+2 t+5\right] .
$$

For $f \in F$, we have

$$
|f| \leq \max \left(\frac{|x|^{5}}{2\left(|x|^{5}+1\right)}+5 t^{3}+4, \frac{|x|}{|x|+1}+2 t+5\right) \leq 19 / 2, \quad x \in \mathbb{R}
$$

Thus,

$$
\|F(t, x)\|_{\mathcal{P}}:=\sup \{|y|: y \in F(t, x)\} \leq 19 / 2=p(t) \psi(\|x\|), \quad x \in \mathbb{R}
$$

with $p(t)=1, \psi(\|x\|)=19 / 2$. Further using the condition

$$
\frac{M}{\frac{\|p\|}{6}\left[1+|\vartheta|\left\{1+\sum_{i=1}^{n-2} \alpha_{i} \frac{\eta_{i}^{4}-\zeta_{i}^{4}}{4}\right\}\right]}>1
$$

we find that $M>2.256269$. This shows that all the assumptions of Theorem 4.8 are satisfied. In consequence, we conclude that there exists at least one solution for the problem (4.3).

\section{Conclusions}

In this paper, we have discussed the existence of solutions for third-order single-valued and multi-valued boundary value problems involving anti-periodic type integral boundary 
conditions and multi-strip boundary conditions. The existence results for single-valued case rely on the standard tools of fixed point theory such as the Leray-Schauder nonlinear alternative, the Banach contraction mapping principle, and fixed point theorems due to Krasnoselskii, and Boyd and Wong, while the existence of solutions for multi-valued case is based on nonlinear alternative of Leray-Schauder type, a selection theorem due to Bressan and Colombo, and Covitz and Nadler's result for contractive multi-valued maps. The results are well illustrated with the aid of several examples. It is interesting to note that the results for problem (1.1) reduce to the ones for a third-order anti-periodic boundary value problem if we take $\mu_{1}=\mu_{2}=\mu_{3}=0$ and $\lambda_{1}=\lambda_{2}=\lambda_{3}=-1$. Further, letting $\lambda_{1}=\lambda_{2}=\lambda_{3}=0$ in problem (1.1) yields the results for a problem with initial-integral boundary conditions. On the other hand, one can notice that arbitrary many strips of finite length are involved in problems (1.2) and (1.3). This consideration is quite flexible as it helps to fix the number of strips as well as their lengths. The problems (1.2) and (1.3) can also be regarded as (2n-2)point nonlocal nonlinear boundary value problems. Furthermore, $\forall \alpha_{i}=0(i=1, \ldots, n-2)$, the results for problems (1.2) and (1.3) correspond to the one with boundary conditions: $x(0)=x^{\prime}(0)=x(1)=0$. In a nutshell, we conclude that the results reported in this study significantly contribute to the existing theory of third-order boundary value problems.

Competing interests

The authors declare that they have no competing interests.

\section{Authors' contributions}

Each of the authors, HHA, SKN, SAA, BA and AA contributed to each part of this work equally and read and approved the final version of the manuscript.

\section{Author details}

'Department of Mathematics, Faculty of Science, King Abdulaziz University, P.O. Box 80203, Jeddah, 21589, Saudi Arabia.

${ }^{2}$ Department of Mathematics, University of loannina, loannina, 451 10, Greece.

\section{Acknowledgements}

This project was funded by the Deanship of Scientific Research (DSR), King Abdulaziz University under grant no. 9/34/GR. The authors, therefore, acknowledge with thanks DSR technical and financial support.

Accepted: 20 December 2014 Published online: 11 February 2015

\section{References}

1. Bressan, A: Hyperbolic Systems of Conservation Laws. The One-Dimensional Cauchy Problem. Oxford University Press, London (2000)

2. Akyildiz, FT, Bellout, H, Vajravelu, K, Van Gorder, RA: Existence results for third order nonlinear boundary value problems arising in nano boundary layer fluid flows over stretching surfaces. Nonlinear Anal., Real World Appl. 12, 2919-2930 (2011)

3. Ahmad, B, Nieto, JJ, Alsaedi, A, El-Shahed, M: A study of nonlinear Langevin equation involving two fractional orders in different intervals. Nonlinear Anal., Real World Appl. 13, 599-606 (2012)

4. Polyanin, AD, Zaitsev, VF: Handbook of Nonlinear Partial Differential Equations. Chapman \& Hall/CRC, Boca Raton (2004)

5. Grossinho, MR, Minhos, FM: Existence result for some third order separated boundary value problems. Nonlinear Anal. 47, 2407-2418 (2001)

6. Yao, Q, Feng, Y: The existence of solution for a third-order two-point boundary value problem. Appl. Math. Lett. 15, 227-232 (2002)

7. Anderson, D: Green's function for a third-order generalized right focal problem. J. Math. Anal. Appl. 288, 1-14 (2003)

8. Bai, Z, Fei, X: Existence of triple positive solutions for a third order generalized right focal problem. Math. Inequal. Appl. 9, 437-444 (2006)

9. Clark, S, Henderson, J: Uniqueness implies existence and uniqueness criterion for non local boundary value problems for third-order differential equations. Proc. Am. Math. Soc. 134, 3363-3372 (2006)

10. Guo, L, Sun, J, Zhao, Y: Existence of positive solution for nonlinear third-order three-point boundary value problem. Nonlinear Anal. 68(10), 3151-3158 (2008)

11. Webb, JRL, Infante, G, Franco, D: Positive solutions of nonlinear fourth order boundary value problems with local and nonlocal boundary conditions. Proc. R. Soc. Edinb., Sect. A 138, 427-446 (2008)

12. Minhos, FM: On some third order nonlinear boundary value problems: existence, location and multiplicity results. J. Math. Anal. Appl. 339, 1342-1353 (2008)

13. Graef, JR, Yang, B: Positive solutions of a third order nonlocal boundary value problem. Discrete Contin. Dyn. Syst., Ser. S 1, 89-97 (2008) 
14. Graef, JR, Kong, L: Positive solutions for third order semipositone boundary value problems. Appl. Math. Lett. 22, 1154-1160 (2009)

15. Graef, JR, Webb, JRL: Third order boundary value problems with nonlocal boundary conditions. Nonlinear Anal. 71, 1542-1551 (2009)

16. Sun, Y: Positive solutions for third-order three-point nonhomogeneous boundary value problems. Appl. Math. Lett. 22, 45-51 (2009)

17. Boucherif, A, Bouguima, SM, Al-Malki, N, Benbouziane, Z: Third order differential equations with integral boundary conditions. Nonlinear Anal. 71, 1736-1743 (2009)

18. Formaggia, L, Nobile, F, Quarteroni, A, Veneziani, A: Multiscale modelling of the circulatory system: a preliminary analysis. Comput. Vis. Sci. 2, 75-83 (1999)

19. Taylor, C, Hughes, T, Zarins, C: Finite element modeling of blood flow in arteries. Comput. Methods Appl. Mech. Eng $158,155-196$ (1998)

20. Womersley, JR: Method for the calculation of velocity, rate of flow and viscous drag in arteries when the pressure gradient is known. J. Physiol. 127, 553-563 (1955)

21. Nicoud, F, Schfonfeld, T: Integral boundary conditions for unsteady biomedical CFD applications. Int. J. Numer. Methods Fluids 40, 457-465 (2002)

22. Cannon, JR: The solution of the heat equation subject to the specification of energy. Q. Appl. Math. 21(2), 155-160 (1963)

23. Ionkin, NI: The solution of a certain boundary value problem of the theory of heat conduction with a nonclassical boundary condition. Differ. Uravn. 13, 294-304 (1977) (in Russian)

24. Chegis, RY: Numerical solution of a heat conduction problem with an integral boundary condition. Liet. Mat. Rink. 24 209-215 (1984)

25. Yurchuk, NI: A mixed problem with an integral condition for some parabolic equations. Differ. Uravn. 22, 2117-2126 (1986) (in Russian)

26. Kartynnik, AV: A three-point mixed problem with an integral condition with respect to the space variable for second-order parabolic equations. Differ. Equ. 26, 1160-1166 (1990)

27. Bouziani, A, Benouar, NE: Mixed problem with integral conditions for a third order parabolic equation. Kobe J. Math. $15,47-58(1998)$

28. Denche, M, Marhoune, AL: Mixed problem with integral boundary condition for a high order mixed type partial differential equation. J. Appl. Math. Stoch. Anal. 16, 69-79 (2003)

29. Infante, G: Eigenvalues and positive solutions of ODEs involving integral boundary conditions. Discrete Contin. Dyn. Syst. suppl., 436-442 (2005)

30. Yang, Z: Positive solutions of a second order integral boundary value problem. J. Math. Anal. Appl. 321, 751-765 (2006)

31. Ahmad, B, Alsaedi, A, Alghamdi, B: Analytic approximation of solutions of the forced Duffing equation with integral boundary conditions. Nonlinear Anal., Real World Appl. 9, 1727-1740 (2008)

32. Boucherif, A: Second-order boundary value problems with integral boundary conditions. Nonlinear Anal. 70, 364-371 (2009)

33. Alsaedi, A, Ahmad, B: Existence and analytic approximation of solutions of Duffing type nonlinear integro-differential equation with integral boundary conditions. J. Inequal. Appl. 2009, Article ID 193169 (2009)

34. Ahmad, B, Alsaedi, A: Existence of approximate solutions of the forced Duffing equation with discontinuous type integral boundary conditions. Nonlinear Anal.. Real World Appl. 10, 358-367 (2009)

35. Agarwal, RP, O'Regan, D, Wong, PJY: Positive Solutions of Differential, Difference, and Integral Equations. Kluwer Academic, Boston (1999)

36. Guo, D, Lakshmikantham, V: Nonlinear Problems in Abstract Cones. Academic Press, San Diego (1988)

\section{Submit your manuscript to a SpringerOpen ${ }^{\circ}$ journal and benefit from:}

- Convenient online submission

Rigorous peer review

- Immediate publication on acceptance

- Open access: articles freely available online

- High visibility within the field

- Retaining the copyright to your article 